\title{
QUANTIZATION OF PSEUDO-DIFFERENTIAL OPERATORS ON THE TORUS
}

\author{
MICHAEL RUZHANSKY AND VILLE TURUNEN
}

\begin{abstract}
Pseudo-differential and Fourier series operators on the torus $\mathbb{T}^{n}=$ $(\mathbb{R} / 2 \pi \mathbb{Z})^{n}$ are analyzed by using global representations by Fourier series instead of local representations in coordinate charts. Toroidal symbols are investigated and the correspondence between toroidal and Euclidean symbols of pseudo-differential operators is established. Periodization of operators and hyperbolic partial differential equations is discussed. Fourier series operators, which are analogues of Fourier integral operators on the torus, are introduced, and formulae for their compositions with pseudo-differential operators are derived. It is shown that pseudo-differential and Fourier series operators are bounded on $L^{2}$ under certain conditions on their phases and amplitudes.
\end{abstract}

\section{Contents}

1. Introduction

2. Preliminaries

3. Difference calculus and discrete Taylor's theorem

4. Pseudo-differential operators and toroidal symbols

5. Extension of toroidal symbols

6. Periodization of pseudo-differential operators

7. Toroidal wave front sets

8. Calculus of Fourier series operators

9. Conditions for $L^{2}$-boundedness

10. Applications to hyperbolic equations

References

\section{INTRODUCTION}

In this paper we investigate a global quantization of pseudo-differential operators on the torus. For this, we develop necessary elements of the toroidal microlocal analysis. Using the toroidal Fourier transform (Fourier series) we can quantize the usual classes of pseudo-differential operators yielding a full symbol of an operator on the torus. The main difference with the (Kohn-Nirenberg) quantization in Hörmander's symbol classes $S_{\rho, \delta}^{m}\left(\mathbb{T}^{n} \times \mathbb{R}^{n}\right)$ (which we will often call Euclidean quantization to emphasize

Date: June 4, 2018.

1991 Mathematics Subject Classification. Primary 58J40; Secondary 35S05, 35S30, 42B05.

Key words and phrases. Pseudo-differential operators, torus, Fourier series, microlocal analysis, Fourier integral operators.

The first author was supported in part by the JSPS Invitational Research Fellowship. 
the difference) is that the toroidal symbol belongs to the corresponding symbol class $S_{\rho, \delta}^{m}\left(\mathbb{T}^{n} \times \mathbb{Z}^{n}\right)$ with frequency variable $\xi \in \mathbb{Z}^{n}$ in the integer lattice; this quantization will be called the toroidal quantization. We will analyze the relation between these symbol classes and between corresponding pseudo-differential operators.

We will also investigate the corresponding toroidal version of Fourier integral operators. To distinguish them from those defined using the Euclidean Fourier transform, we will call them Fourier series operators. The use of Fourier series will allow us to obtain a global representation of these operators, thus making trivial a number of topological obstructions known in the standard theory of Fourier integral operators on manifolds. We will prepare the machinery and describe how it can be further used in the calculus of Fourier series operators and applications to hyperbolic partial differential equations. In fact, the form of the required discrete calculus is not a-priori clear. For example, composition formulae for pseudo-differential operators rely on the Taylor expansion of symbols which are now defined on the discrete lattice $\mathbb{Z}^{n}$. Thus, we develop the corresponding versions of the periodic and discrete analysis which are still quite similar to formulations in the standard Euclidean theory. In particular, this will include the analysis of differences and some elements of the microlocal analysis such as toroidal wave front sets, etc. We study pseudo-differential operators in detail giving an explicit relation between Euclidean and toroidal symbols. Moreover, we will describe how this relation can be used to relate Euclidean and toroidal quantizations of the the same operators using a periodization operator that we analyze for this purpose.

However, our analysis will reveal certain limitations for the development of an equivalent full theory for Fourier series operators. The main difference with pseudodifferential operators is in the behaviour of the wave front set under the action of operators. In the case of an pseudo-differential operator, the wave front set does not move and so we remain in the space-frequency space $\mathbb{T}^{n} \times \mathbb{Z}^{n}$. In the case of a Fourier series operator, their integral kernel may have singularities away from the diagonal in $\mathbb{T}^{n} \times \mathbb{T}^{n}$, which means that under the flow the wave front set may no longer be a transformation of $\mathbb{T}^{n} \times \mathbb{Z}^{n}$. Nevertheless, we will be able to resolve this issue in the composition formulae by using (unique modulo smoothing) extensions of toroidal symbols.

It was realized already in the 1970s that on the torus one can study pseudodifferential operators globally using Fourier series expansions, in analogy to the Euclidean pseudo-differential calculus. These periodic pseudo-differential operators were treated e.g. by Agranovich [1, 2]. Contributions have been made by many authors, e.g. by Amosov [3], Elschner [11], McLean [16], Melo [17], Prössdorf and Schneider [18], Saranen and Wendland [26], Turunen [31], Turunen and Vainikko [32], Vainikko and Lifanov [33, 34, and others. However, most of these papers deal with onedimensional cases or with classes of operators rather than with classes of symbols. In this paper we in particular develop the symbolic analysis of pseudo-differential operators on the torus thus closely relating Euclidean and toroidal quantizations of the same classes of operators.

We note that the use of operators which are discrete in the frequency variable allows one to weaken regularity assumptions on symbols with respect to $\xi$-variables. Symbols with low regularity in $x$ have been under intensive study for many years, e.g. see 
Kumano-go and Nagase [15], Sugimoto [29], Boulkhemair [4], Garello and Morando [12], and many other contributions. However, in these papers one assumes symbols to be smooth or sufficiently regular in $\xi$. The discrete approach in this paper will allow us to reduce or to completely remove the regularity assumptions with respect to $\xi$. For example, no regularity with respect to $\xi$ is assumed for $L^{2}$ estimates, and for elements of the calculus. We note that a notion of pseudo-differential operators with low regularity in $\xi$ can still be introduced, as was done e.g. by Sjöstrand [27], or with symbols in more general modulation spaces, see e.g. Gröchenig [13]. Although such operators form an algebra, no explicit symbolic calculus of such operators is available. In this respect the toroidal quantization has an advantage of looking only at discrete values of $\xi$, thus removing the regularity issue altogether, and yielding the symbolic calculus.

We note that the approach developed in this paper may be generalised to other (non-commutative) Lie groups. Thus, we will develop global quantizations of pseudodifferential operators on $\mathbb{S}^{3}$ in 23 ] by identifying it with group $\mathrm{SU}(2)$ via the quaternionic product in $\mathbb{R}^{4}$. Moreover, in [24] we will also develop global quantizations of pseudo-differential operators on arbitrary compact Lie groups and on certain homogeneous spaces without resorting to local coordinate charts. We note that this analysis is rather different from the one developed by Taylor in [30] because of the obtained descriptions of symbol classes and their transformations. In any case, the relation between compact Lie groups and $\mathbb{R}^{n}$ is most transparent in the case of the torus and the utilisation of this relation brings considerable simplifications in the analysis of this paper compared to that in [23] and [24], which rely heavily on the representation theory. Some results proved in this paper were announced in [22].

We fix the notation for the torus as $\mathbb{T}^{n}=(\mathbb{R} / 2 \pi \mathbb{Z})^{n}$. Often we may identify $\mathbb{T}^{n}$ with the cube $[0,2 \pi)^{n} \subset \mathbb{R}^{n}$ (or $[-\pi, \pi)^{n}$ ), where we identify the measure on the torus with the restriction of the Euclidean measure on the cube. Functions on $\mathbb{T}^{n}$ can be thought as those functions on $\mathbb{R}^{n}$ that are $2 \pi$-periodic in each of the coordinate directions. We will often simply say that such functions are $2 \pi$-periodic (instead of $2 \pi \mathbb{Z}^{n}$-periodic)

For $N \in \mathbb{N}$ we will also use the notation $N \mathbb{T}^{n}=(\mathbb{R} / 2 \pi N \mathbb{Z})^{n}$ and call this the $N$-inflated torus, or simply an inflated torus if the value of $N$ is not of importance. One of the reasons to use large $N$ in this analysis is that if one wants to embed a compactly supported problem in $\mathbb{R}^{n}$ into a torus, one may need to choose $N$ large enough for technical simplifications. We will briefly discuss these issues.

One particular application discussed in this paper will be to hyperbolic equations on the torus. In particular, the developed analysis can be applied if we embed certain problems in $\mathbb{R}^{n}$ into the torus. In general, we can observe that due to the finite propagation speed of singularities in hyperbolic equations we can usually cut-off the equation and the Cauchy data for large $x$ in the local analysis of singularities of solutions for bounded times. Then we can embed the problem into $\mathbb{T}^{n}$ using rescaling (or into the $N$-inflated torus $N \mathbb{T}^{n}$ without rescaling) in order to apply the periodic analysis developed in this paper. One of the advantages of this procedure is that since phases and amplitudes now are evaluated only at $\xi \in \mathbb{Z}^{n}$ one can apply this also for problems with low regularity in $\xi$. This type of application is particularly important for weakly hyperbolic equations or systems with variable multiplicities when at points 
of multiplicities characteristics become irregular. If the principal part has constant coefficients then the loss of regularity occurs only in $\xi$, so techniques developed in this paper can be applied. Such applications to systems will appear elsewhere and in this paper we shall briefly discuss only the case of scalar equations.

We also note that this approach to the representation of solutions is related to the work of Bourgain [5, 6, 7] on Strichartz estimates for solutions to Schrödinger equations on the torus $\mathbb{T}^{n}$. Bourgain used the Fourier series representation

$$
u(t, x)=\mathrm{e}^{\mathrm{i} t \Delta} f(x)=\sum_{\xi \in \mathbb{Z}^{n}} \mathrm{e}^{\mathrm{i}\left(x \cdot \xi-t|\xi|^{2}\right)} \widehat{f}(\xi)
$$

which shows in addition that the solution is periodic in time. This representation allowed him to prove, for example, the equality $\|u\|_{L^{4}\left(\mathbb{T} \times \mathbb{T}^{n}\right)}^{4}=\left\|u^{2}\right\|_{L^{2}\left(\mathbb{T} \times \mathbb{T}^{n}\right)}^{2}$ leading to the corresponding Strichartz estimates and global well-posedness results for nonlinear equations (while dispersive estimates fail even locally in time). In Section 10 we discuss solutions to hyperbolic equations with variable coefficients but leaving corresponding nonlinear applications outside the scope of this paper.

Overall, the analysis developed in this paper lays down a foundation for tackling a variety of global problems on the torus with the use of the full toroidal symbols (questions like global hypoellipticity, global solvability, estimates for pseudo-differential operators, Fefferman-Phong inequality, etc.), and for applying these techniques to partial differential equations. Again, we leave further applications outside the scope of this paper.

In Section 2 we fix the notation used throughout the paper. In Section 3 we develop the necessary machinery for the discrete calculus, in particular we prove the Taylor expansion formula on the lattice $\mathbb{Z}^{n}$ giving an estimate for the remainder. Section 4 is devoted to the notion and main properties of toroidal symbols together with formulae for their calculus. In Section 5 we establish the relation between Euclidean and toroidal symbols and in Section 6 the relation between operators with Euclidean and toroidal quantizations. In Section 7 we prove basic results about toroidal wave front sets. Section 8 introduces Fourier series operators and establishes composition formulae between Fourier series and pseudo-differential operators. In Section 9 we prove results on the $L^{2}$-boundedness of pseudo-differential and Fourier series operators. Finally, Section 10 is devoted to an application to hyperbolic equations showing a way how to embed such equations into the torus by periodization.

We will use the following notation in the paper. Triangles $\triangle$ and $\bar{\triangle}$ will denote the forward and backward difference operators, respectively. The Laplacian will be denoted by $\mathscr{L}$. The Dirac delta at $x$ will be denoted by $\delta_{x}(y)$ and the Kronecker delta at $\xi$ will be denoted by $\delta_{\xi, \eta}$. We will use the standard notation for multi-indices $\alpha \in \mathbb{N}_{0}^{n}$, with $\mathbb{N}_{0}=\{0\} \cup \mathbb{N}$, for example denoting $|\alpha|:=\sum_{j=1}^{n}\left|\alpha_{j}\right|, \alpha !=\alpha_{1} ! \cdots \alpha_{n}$ !, $\partial_{x}^{\alpha}=\partial_{x_{1}}^{\alpha_{1}} \cdots \partial_{x_{n}}^{\alpha_{n}}$, etc. We will also use the standard notation $D_{x_{j}}=-\mathrm{i} \partial_{x_{j}}=\frac{1}{\mathrm{i}} \frac{\partial}{\partial x_{j}}$, where $\mathrm{i}=\sqrt{-1}$. We will write $a \in C^{\infty}\left(\mathbb{T}^{n} \times \mathbb{Z}^{n}\right)$ when function $a(\cdot, \xi)$ is smooth on $\mathbb{T}^{n}$ for all $\xi \in \mathbb{Z}^{n}$. For symbol classes $S_{\rho, \delta}^{m}$, we will often write simply $S^{m}$ for the class $S_{1,0}^{m}$. For $\xi \in \mathbb{R}^{n}$, we will write $\langle\xi\rangle=\left(1+|\xi|^{2}\right)^{1 / 2}$. To avoid keeping track of constants in integrals we will normalise the measure by defining $\mathrm{d} x=(2 \pi)^{-n} \mathrm{~d} x$. Constants 
will be usually denoted by $C$ (sometimes with subscripts), and their values may differ on different occasions, even when appearing in subsequent estimates.

\section{Preliminaries}

In this section we will introduce some notation which will be frequently used in the sequel. Let $\mathcal{S}\left(\mathbb{R}^{n}\right)$ denote the space of the Schwartz test functions, and let $\mathcal{S}^{\prime}\left(\mathbb{R}^{n}\right)$ be its dual, i.e. the space of the tempered distributions. The Dirac delta comb $\delta_{\mathbb{Z}^{n}}: \mathcal{S}\left(\mathbb{R}^{n}\right) \rightarrow \mathbb{C}$ is defined by

$$
\left\langle\delta_{\mathbb{Z}^{n}}, \varphi\right\rangle:=\sum_{x \in \mathbb{Z}^{n}} \varphi(x),
$$

and the sum here is absolutely convergent.

Let $\mathcal{S}\left(\mathbb{Z}^{n}\right)$ denote the space of rapidly decaying functions $\mathbb{Z}^{n} \rightarrow \mathbb{C}$. That is, $\varphi \in$ $\mathcal{S}\left(\mathbb{Z}^{n}\right)$ if for any $M<\infty$ there exists a constant $C_{\varphi, M}$ such that $|\varphi(\xi)| \leq C_{\varphi, M}\langle\xi\rangle^{-M}$ holds for all $\xi \in \mathbb{Z}^{n}$. The topology on $\mathcal{S}\left(\mathbb{Z}^{n}\right)$ is given by the seminorms $p_{k}$, where $k \in \mathbb{N}_{0}$ and $p_{k}(\varphi):=\sup _{\xi \in \mathbb{Z}^{n}}\langle\xi\rangle^{k}|\varphi(\xi)|$. One can show that the continuous linear functionals on $\mathcal{S}\left(\mathbb{Z}^{n}\right)$ are of the form

$$
\varphi \mapsto\langle u, \varphi\rangle:=\sum_{\xi \in \mathbb{Z}^{n}} u(\xi) \varphi(\xi),
$$

where functions $u: \mathbb{Z}^{n} \rightarrow \mathbb{C}$ grow at most polynomially at infinity, i.e. there exist constants $M<\infty$ and $C_{u, M}$ such that

$$
|u(\xi)| \leq C_{u, M}\langle\xi\rangle^{M}
$$

holds for all $\xi \in \mathbb{Z}^{n}$. Such distributions $u: \mathbb{Z}^{n} \rightarrow \mathbb{C}$ form the space $\mathcal{S}^{\prime}\left(\mathbb{Z}^{n}\right)$.

Let $\mathscr{F}_{\mathbb{R}^{n}}: \mathcal{S}\left(\mathbb{R}^{n}\right) \rightarrow \mathcal{S}\left(\mathbb{R}^{n}\right)$ be the Euclidean Fourier transform defined by

$$
\left(\mathscr{F}_{\mathbb{R}^{n}} f\right)(\xi):=\int_{\mathbb{R}^{n}} \mathrm{e}^{-\mathrm{i} x \cdot \xi} f(x) \mathrm{d} x
$$

where đ $x=(2 \pi)^{-n} \mathrm{~d} x$. Mapping $\mathscr{F}_{\mathbb{R}^{n}}: \mathcal{S}\left(\mathbb{R}^{n}\right) \rightarrow \mathcal{S}\left(\mathbb{R}^{n}\right)$ is a bijection, and its inverse $\mathscr{F}_{\mathbb{R}^{n}}^{-1}$ is given by

$$
f(x)=\int_{\mathbb{R}^{n}} \mathrm{e}^{\mathrm{i} x \cdot \xi}\left(\mathscr{F}_{\mathbb{R}^{n}} f\right)(\xi) \mathrm{d} \xi .
$$

As it is well-known, this Fourier transform can be uniquely extended to $\mathscr{F}_{\mathbb{R}^{n}}$ : $\mathcal{S}^{\prime}\left(\mathbb{R}^{n}\right) \rightarrow \mathcal{S}^{\prime}\left(\mathbb{R}^{n}\right)$ by duality.

Let $\mathscr{F}_{\mathbb{T}^{n}}: C^{\infty}\left(\mathbb{T}^{n}\right) \rightarrow \mathcal{S}\left(\mathbb{Z}^{n}\right)$ be the toroidal Fourier transform defined by

$$
\left(\mathscr{F}_{\mathbb{T}^{n}} f\right)(\xi):=\int_{\mathbb{T}^{n}} \mathrm{e}^{-\mathrm{i} x \cdot \xi} f(x) \mathrm{d} x
$$

where $\mathrm{d} x=(2 \pi)^{-n} \mathrm{~d} x$. Then $\mathscr{F}_{\mathbb{T}^{n}}$ is a bijection and its inverse $\mathscr{F}_{\mathbb{T}^{n}}^{-1}: \mathcal{S}\left(\mathbb{Z}^{n}\right) \rightarrow$ $C^{\infty}\left(\mathbb{T}^{n}\right)$ is given by

$$
\left(\mathscr{F}_{\mathbb{T}^{n}}^{-1} g\right)(x)=\sum_{\xi \in \mathbb{Z}^{n}} \mathrm{e}^{\mathrm{i} x \cdot \xi} g(\xi)
$$


so that $f(x)=\sum_{\xi \in \mathbb{Z}^{n}} \mathrm{e}^{\mathrm{i} x \cdot \xi}\left(\mathscr{F}_{\mathbb{T}^{n}} f\right)(\xi)$. By dualising $\mathscr{F}_{\mathbb{T}^{n}}^{-1}: \mathcal{S}\left(\mathbb{Z}^{n}\right) \rightarrow C^{\infty}\left(\mathbb{T}^{n}\right)$, the Fourier transform is extended uniquely to mapping $\mathscr{F}_{\mathbb{T}^{n}}: \mathcal{D}^{\prime}\left(\mathbb{T}^{n}\right) \rightarrow \mathcal{S}^{\prime}\left(\mathbb{Z}^{n}\right)$ by the formula

$$
\left\langle\mathscr{F}_{\mathbb{T}^{n}} u, \varphi\right\rangle:=\left\langle u, \iota \circ \mathscr{F}_{\mathbb{T}^{n}}^{-1} \varphi\right\rangle,
$$

where $u \in \mathcal{D}^{\prime}\left(\mathbb{T}^{n}\right), \varphi \in \mathcal{S}\left(\mathbb{Z}^{n}\right)$, and $\iota$ is defined by $(\iota \circ \psi)(x)=\psi(-x)$. One can easily check that extension (2.2) when restricted to $C^{\infty}\left(\mathbb{T}^{n}\right)$, is compatible with the definition (2.1). Here, inclusion $C^{\infty}\left(\mathbb{T}^{n}\right) \subset \mathcal{D}^{\prime}\left(\mathbb{T}^{n}\right)$ is interpreted by $\langle u, \varphi\rangle=$ $\int_{\mathbb{T}^{n}} u(x) \varphi(x)$ đ $x$.

It can be also easily seen that the Fourier analysis on the $N$-inflated torus $N \mathbb{T}^{n}=$ $(\mathbb{R} / 2 \pi N \mathbb{Z})^{n}$ can be obtained from the Fourier analysis of the standard torus $\mathbb{T}^{n}$ by changes of variables. We can identify the dual group $\widehat{N \mathbb{T}^{n}}$ of $N \mathbb{T}^{n}$ with $\frac{1}{N} \mathbb{Z}^{n}$. On $N \mathbb{T}^{n}$, we can define the Fourier transform $\mathscr{F}_{N \mathbb{T}^{n}}: C^{\infty}\left(N \mathbb{T}^{n}\right) \rightarrow \mathcal{S}\left(\frac{1}{N} \mathbb{Z}^{n}\right)$ by

$$
\left(\mathscr{F}_{N \mathbb{T}^{n}} g\right)(\eta):=\int_{N \mathbb{T}^{n}} g(y) \mathrm{e}^{-\mathrm{i} y \cdot \eta} \text { đ } y, \quad \eta \in \frac{1}{N} \mathbb{Z}^{n},
$$

where $\mathrm{d} y=(2 \pi)^{-n} \mathrm{~d} y$. Let us denote $g_{N}(x)=g(N x)$. Then it is easy to see that we have the relation

$$
\mathscr{F}_{N \mathbb{T}^{n}} g(\eta)=N^{n}\left(\mathscr{F}_{\mathbb{T}^{n}} g_{N}\right)(N \eta), \quad \eta \in \frac{1}{N} \mathbb{Z}^{n} .
$$

\section{Difference CAlculus AND Discrete TAYlor's TheOREM}

In this section we develop the discrete calculus which will be needed in the sequel. In particular, we will formulate and prove a discrete version of the Taylor expansion formula on the lattice $\mathbb{Z}^{n}$.

Let $\sigma: \mathbb{Z}^{n} \rightarrow \mathbb{C}$. Let $e_{j} \in \mathbb{N}^{n},\left(e_{j}\right)_{j}=1$ and $\left(e_{j}\right)_{i}=0$ if $i \neq j$. Define the partial difference operator $\triangle_{\xi_{j}}$ by

$$
\triangle_{\xi_{j}} \sigma(\xi):=\sigma\left(\xi+e_{j}\right)-\sigma(\xi),
$$

and define

$$
\triangle_{\xi}^{\alpha}=\triangle_{\xi_{1}}^{\alpha_{1}} \cdots \triangle_{\xi_{n}}^{\alpha_{n}}
$$

for $\alpha \in \mathbb{N}_{0}^{n}$. It can be easily checked that these difference operators commute, i.e. that $\triangle_{\xi}^{\alpha} \triangle_{\xi}^{\beta}=\triangle_{\xi}^{\beta} \triangle_{\xi}^{\alpha}$ for all multi-indices $\alpha, \beta \in \mathbb{N}_{0}^{n}$. Let us summarize properties of differences:

Proposition 3.1. We have

$$
\triangle_{\xi}^{\alpha} \sigma(\xi)=\sum_{\beta \leq \alpha}(-1)^{|\alpha-\beta|}\left(\begin{array}{c}
\alpha \\
\beta
\end{array}\right) \sigma(\xi+\beta)
$$

Let $\phi, \psi: \mathbb{Z}^{n} \rightarrow \mathbb{C}$. Then we have the Leibnitz formula for differences:

$$
\triangle_{\xi}^{\alpha}(\phi \psi)(\xi)=\sum_{\beta \leq \alpha}\left(\begin{array}{l}
\alpha \\
\beta
\end{array}\right)\left(\triangle_{\xi}^{\beta} \phi(\xi)\right)\left(\triangle_{\xi}^{\alpha-\beta} \psi\right)(\xi+\beta) .
$$

Also, the "summation by parts" is given by

$$
\sum_{\xi \in \mathbb{Z}^{n}} \phi(\xi)\left(\triangle_{\xi}^{\alpha} \psi\right)(\xi)=(-1)^{|\alpha|} \sum_{\xi \in \mathbb{Z}^{n}}\left(\bar{\triangle}_{\xi}^{\alpha} \phi\right)(\xi) \psi(\xi)
$$


where $\left(\overline{\triangle_{\xi_{j}}} \phi\right)(\xi)=\phi(\xi)-\phi\left(\xi-e_{j}\right)$, with the iterative definition for higher order differences. In (3.1) we assume that both series are absolutely convergent.

Proposition 3.1 can be proved by induction and we omit the proof. For $\theta \in \mathbb{Z}^{n}$ and $\alpha \in \mathbb{N}_{0}^{n}$, we define $\theta^{(\alpha)}=\theta_{1}^{\left(\alpha_{1}\right)} \cdots \theta_{n}^{\left(\alpha_{n}\right)}$, where $\theta_{j}^{(0)}=1$ and

$$
\theta_{j}^{(k+1)}=\theta_{j}^{(k)}\left(\theta_{j}-k\right)=\theta_{j}\left(\theta_{j}-1\right) \ldots\left(\theta_{j}-k\right) .
$$

Then we have $\triangle_{\theta}^{\gamma} \theta^{(\alpha)}=\alpha^{(\gamma)} \theta^{(\alpha-\gamma)}$, in analogy to the Euclidean case when $\partial_{\theta}^{\gamma} \theta^{\alpha}=$ $\alpha^{(\gamma)} \theta^{\alpha-\gamma}$.

For $b \geq 0$, let us denote

$$
I_{k}^{b}:=\sum_{0 \leq k<b} \text { and } I_{k}^{-b}:=-\sum_{-b \leq k<0} .
$$

One can think of $I_{\xi}^{\theta} \cdots$ as a discrete version of the one-dimensional integral $\int_{0}^{\theta} \cdots \mathrm{d} \xi$; in this discrete context, the difference $\triangle_{\xi}$ takes the role of the differential operator $\mathrm{d} / \mathrm{d} \xi$.

In the sequel, we adopt the notational conventions

$$
I_{k_{1}}^{\theta} I_{k_{2}}^{k_{1}} \cdots I_{k_{\alpha}}^{k_{\alpha-1}} 1= \begin{cases}1, & \text { if } \alpha=0 \\ I_{k_{1}}^{\theta} 1, & \text { if } \alpha=1 \\ I_{k_{1}}^{\theta} I_{k_{2}}^{k_{1}} 1, & \text { if } \alpha=2\end{cases}
$$

and so on.

Lemma 3.2. If $\theta \in \mathbb{Z}$ and $\alpha \in \mathbb{N}_{0}$ then

$$
I_{k_{1}}^{\theta} I_{k_{2}}^{k_{1}} \cdots I_{k_{\alpha}}^{k_{\alpha-1}} 1=\frac{1}{\alpha !} \theta^{(\alpha)} .
$$

Proof. We observe simple equalities $k^{(0)} \equiv 1, \triangle_{k} k^{(i)}=i k^{(i-1)}$ and $I_{k}^{b} \triangle_{k} k^{(i)}=b^{(i)}$, from which (3.4) follows by induction.

We note that Lemma 3.2 can be viewed as a discrete trivial version of the fundamental theorem of calculus: $\int_{0}^{\theta} f^{\prime}(\xi) \mathrm{d} \xi=f(\theta)-f(0)$ for smooth enough $f: \mathbb{R} \rightarrow \mathbb{C}$ corresponds to $I_{\xi}^{\theta} \triangle_{\xi} f(\xi)=f(\theta)-f(0)$ for $f: \mathbb{Z} \rightarrow \mathbb{C}$. Lemma 3.2 immediately implies its multidimensional version:

Corollary 3.3. If $\theta \in \mathbb{Z}^{n}$ and $\alpha \in \mathbb{N}_{0}^{n}$ then

$$
\prod_{j=1}^{n} I_{k(j, 1)}^{\theta_{j}} I_{k(j, 2)}^{k(j, 1)} \cdots I_{k\left(j, \alpha_{j}\right)}^{k\left(j, \alpha_{j}-1\right)} 1=\frac{1}{\alpha !} \theta^{(\alpha)},
$$

where $\prod_{j=1}^{n} I_{j}$ means $I_{1} I_{2} \cdots I_{n}$, where $I_{j}:=I_{k(j, 1)}^{\theta_{j}} I_{k(j, 2)}^{k(j, 1)} \cdots I_{k\left(j, \alpha_{j}\right)}^{k\left(j, \alpha_{j}-1\right)}$.

Now we give the multidimensional version of the Taylor expansion formula.

Theorem 3.4 (Discrete Taylor series on $\mathbb{Z}^{n}$ ). Let $p: \mathbb{Z}^{n} \rightarrow \mathbb{C}$. Then we can write

$$
p(\xi+\theta)=\sum_{|\alpha|<M} \frac{1}{\alpha !} \theta^{(\alpha)} \triangle_{\xi}^{\alpha} p(\xi)+r_{M}(\xi, \theta),
$$


with remainder $r_{M}(\xi, \theta)$ satisfying

$$
\left|\triangle_{\xi}^{\omega} r_{M}(\xi, \theta)\right| \leq C_{M}\left|\theta^{(\alpha)}\right| \max _{|\alpha|=M, \nu \in Q(\theta)}\left|\triangle_{\xi}^{\alpha+\omega} p(\xi+\nu)\right|,
$$

where $Q(\theta):=\left\{\nu \in \mathbb{Z}^{n}:\left|\nu_{j}\right| \leq\left|\theta_{j}\right|\right.$ for all $\left.j=1, \ldots, n\right\}$.

Proof. For $0 \neq \alpha \in \mathbb{N}_{0}^{n}$, let us denote $m_{\alpha}:=\min \left\{j: \alpha_{j} \neq 0\right\}$. For $\theta \in \mathbb{Z}^{n}$ and $i \in\{1, \ldots, n\}$, let us define $\nu(\theta, i, k) \in \mathbb{Z}^{n}$ by

$$
\nu(\theta, i, k):=\left(\theta_{1}, \ldots, \theta_{i-1}, k, 0, \ldots, 0\right),
$$

i.e.

$$
\nu(\theta, i, k)_{j}= \begin{cases}\theta_{j}, & \text { if } 1 \leq j<i \\ k, & \text { if } j=i \\ 0, & \text { if } i<j \leq n .\end{cases}
$$

We claim that the remainder can be written in the form

$$
r_{M}(\xi, \theta)=\sum_{|\alpha|=M} r_{\alpha}(\xi, \theta)
$$

where for each $\alpha$, we have

$$
r_{\alpha}(\xi, \theta)=\prod_{j=1}^{n} I_{k(j, 1)}^{\theta_{j}} I_{k(j, 2)}^{k(j, 1)} \cdots I_{k\left(j, \alpha_{j}\right)}^{k\left(j, \alpha_{j}-1\right)} \triangle_{\xi}^{\alpha} p\left(\xi+\nu\left(\theta, m_{\alpha}, k\left(m_{\alpha}, \alpha_{m_{\alpha}}\right)\right)\right)
$$

recall (3.3) and (3.5) . The proof of (3.8) is by induction. The first remainder term $r_{1}$ is of the claimed form, since

$$
r_{1}(\xi, \theta)=p(\xi+\theta)-p(\xi)=\sum_{i=1}^{n} r_{e_{i}}(\xi, \theta)
$$

where

$$
r_{e_{i}}(\xi, \theta)=I_{k}^{\theta_{i}} \triangle_{\xi}^{e_{i}} p(\xi+\nu(\theta, i, k)) ;
$$

here $r_{e_{i}}$ is of the form (3.8) for $\alpha=e_{i}, m(\alpha)=i$ and $\alpha_{m_{\alpha}}=1$. So suppose that the claim (3.8) is true up to order $|\alpha|=M$. Then

$$
\begin{aligned}
r_{M+1}(\xi, \theta)= & r_{M}(\xi, \theta)-\sum_{|\alpha|=M} \frac{1}{\alpha !} \theta^{(\alpha)} \triangle_{\xi}^{\alpha} p(\xi) \\
= & \sum_{|\alpha|=M}\left(r_{\alpha}(\xi, \theta)-\frac{1}{\alpha !} \theta^{(\alpha)} \triangle_{\xi}^{\alpha} p(\xi)\right) \\
= & \sum_{|\alpha|=M} \prod_{j=1}^{n} I_{k(j, 1)}^{\theta_{j}} I_{k(j, 2)}^{k(j, 1)} \cdots I_{k\left(j, \alpha_{j}\right)}^{k\left(j, \alpha_{j}-1\right)} \\
& \triangle_{\xi}^{\alpha}\left[p\left(\xi+\nu\left(\theta, m_{\alpha}, k\left(m_{\alpha}, \alpha_{m_{\alpha}}\right)\right)-p(\xi)\right],\right.
\end{aligned}
$$

where we used (3.8) and (3.5) to obtain the last equality. Combining this with the equality

$$
p\left(\xi+\nu\left(\theta, m_{\alpha}, k\right)\right)-p(\xi)=\sum_{i=1}^{m_{\alpha}} I_{\ell}^{\nu\left(\theta, m_{\alpha}, k\right)_{i}} \triangle_{\xi}^{v_{i}} p(\xi+\nu(\theta, i, \ell))
$$


we get

$$
\begin{aligned}
r_{M+1}(\xi, \theta)= & \sum_{|\alpha|=M} \prod_{j=1}^{n} I_{k(j, 1)}^{\theta_{j}} I_{k(j, 2)}^{k(j, 1)} \cdots I_{k\left(j, \alpha_{j}\right)}^{k\left(j, \alpha_{j}-1\right)} \sum_{i=1}^{m_{\alpha}} I_{\ell(i)}^{\nu\left(\theta, m_{\alpha}, k\left(m_{\alpha}, \alpha_{m_{\alpha}}\right)\right)_{i}} \\
& \triangle_{\xi}^{\alpha+e_{i}} p(\xi+\nu(\theta, i, \ell(i))) \\
= & \sum_{|\beta|=M+1} \prod_{j=1}^{n} I_{k(j, 1)}^{\theta_{j}} I_{k(j, 2)}^{k(j, 1)} \cdots I_{k\left(j, \beta_{j}\right)}^{k\left(j, \beta_{j}-1\right)} \triangle_{\xi}^{\beta} p\left(\xi+\nu\left(\theta, m_{\beta}, k\left(m_{\beta}, \beta_{m_{\beta}}\right)\right)\right) ;
\end{aligned}
$$

the last step here is just simple tedious book-keeping. Thus the induction proof of (3.8) is complete. Finally, let us prove estimate (3.6). By (3.8), we obtain

$$
\begin{aligned}
& \left|\triangle_{\xi}^{\omega} r_{M}(\xi, \theta)\right|= \\
= & \left|\sum_{|\alpha|=M} \triangle_{\xi}^{\omega} r_{\alpha}(\xi, \theta)\right| \\
= & \left|\sum_{|\alpha|=M} \prod_{j=1}^{n} I_{k(j, 1)}^{\theta_{j}} I_{k(j, 2)}^{k(j, 1)} \cdots I_{k\left(j, \alpha_{j}\right)}^{k\left(j, \alpha_{j}-1\right)} \triangle_{\xi}^{\alpha+\omega} p\left(\xi+\nu\left(\theta, m_{\alpha}, k\left(m_{\alpha}, \alpha_{m_{\alpha}}\right)\right)\right)\right| \\
\leq & \sum_{|\alpha|=M} \frac{1}{\alpha !}\left|\theta^{(\alpha)}\right| \max _{\nu \in Q(\theta)}\left|\triangle_{\xi}^{\alpha+\omega} p(\xi+\nu)\right|
\end{aligned}
$$

where in the last step we used (3.5). The proof is complete.

Remark 3.5. In Theorem 3.4 we estimated the remainder over the discrete box $Q(\theta)$, but an estimate over a discrete path would have been enough.

\section{Pseudo-Differential operators AND toroidal SyMbOls}

We note that given a continuous linear operator $A: C^{\infty}\left(\mathbb{T}^{n}\right) \rightarrow C^{\infty}\left(\mathbb{T}^{n}\right)$, we can consider its toroidal quantization

$$
A \varphi(x)=\sum_{\xi \in \mathbb{Z}^{n}} \int_{\mathbb{T}^{n}} \mathrm{e}^{\mathrm{i}(x-y) \cdot \xi} \sigma_{A}(x, \xi) f(y) \mathrm{d} y,
$$

where its toroidal symbol $\sigma_{A} \in C^{\infty}\left(\mathbb{T}^{n} \times \mathbb{Z}^{n}\right)$ is uniquely defined by the formula

$$
\sigma_{A}(x, \xi)=\mathrm{e}^{-\mathrm{i} x \cdot \xi} A e_{\xi}(x),
$$

where $e_{\xi}(x):=\mathrm{e}^{\mathrm{i} x \cdot \xi}$. We note that for pseudo-differential operators this would be just another quantization of the same class, see Remark 4.1 for a precise statement.

For $\psi, \varphi \in C^{\infty}\left(\mathbb{T}^{n}\right)$, let $\psi \otimes \varphi \in C^{\infty}\left(\mathbb{T}^{2 n}\right)$ be defined by $(\psi \otimes \varphi)(x, y):=\psi(x) \varphi(y)$. If $A: C^{\infty}\left(\mathbb{T}^{n}\right) \rightarrow \mathcal{D}^{\prime}\left(\mathbb{T}^{n}\right)$ is a continuous linear operator then one can verify that

$$
\left\langle K_{A}, \psi \otimes \varphi\right\rangle:=\langle A \varphi, \psi\rangle
$$

defines the periodic Schwarz distributional kernel $K_{A} \in \mathcal{D}^{\prime}\left(\mathbb{T}^{2 n}\right)$ of operator $A \in$ $\mathcal{L}\left(C^{\infty}\left(\mathbb{T}^{n}\right), \mathcal{D}^{\prime}\left(\mathbb{T}^{n}\right)\right)$; a common informal notation is

$$
A \varphi(x)=\int_{\mathbb{T}^{n}} K_{A}(x, y) \varphi(y) \mathrm{d} y .
$$


The convolution kernel $k_{A} \in \mathcal{D}^{\prime}\left(\mathbb{T}^{2 n}\right)$ of $A$ is related to the Schwartz kernel by $K_{A}(x, y)=k_{A}(x, x-y)$, i.e. we have

$$
A \varphi(x)=\int_{\mathbb{T}^{n}} k_{A}(x, x-y) \varphi(y) \mathrm{d} y
$$

in the sense of distributions. Notice that if $k_{A}(x)(y)=k_{A}(x, y)$ then

$$
\mathscr{F}_{\mathbb{T}^{n}}\left(k_{A}(x)\right)(\xi)=\sigma_{A}(x, \xi) .
$$

Let $m \in \mathbb{R}, 0 \leq \delta, \rho \leq 1$. Then the toroidal symbol class $S_{\rho, \delta}^{m}\left(\mathbb{T}^{n} \times \mathbb{Z}^{n}\right)$ consists of those functions $a(x, \xi)$ which are smooth in $x$ for all $\xi \in \mathbb{Z}^{n}$, and which satisfy

$$
\left|\triangle_{\xi}^{\alpha} \partial_{x}^{\beta} a(x, \xi)\right| \leq C_{a \alpha \beta m}\langle\xi\rangle^{m-\rho|\alpha|+\delta|\beta|}
$$

for every $x \in \mathbb{T}^{n}$, for every $\alpha, \beta \in \mathbb{N}_{0}^{n}$, and for all $\xi \in \mathbb{Z}^{n}$. The class $S_{1,0}^{m}\left(\mathbb{T}^{n} \times \mathbb{Z}^{n}\right)$ will be often denoted by writing simply $S^{m}\left(\mathbb{T}^{n} \times \mathbb{Z}^{n}\right)$.

If $a \in S_{\rho, \delta}^{m}\left(\mathbb{T}^{n} \times \mathbb{Z}^{n}\right)$, we denote by $a(X, D) \in \mathrm{Op} S_{\rho, \delta}^{m}\left(\mathbb{T}^{n} \times \mathbb{Z}^{n}\right)$ the corresponding toroidal pseudo-differential operator defined by

$$
a(X, D) f(x)=\sum_{\xi \in \mathbb{Z}^{n}} \int_{\mathbb{T}^{n}} \mathrm{e}^{\mathrm{i}(x-y) \cdot \xi} a(x, \xi) f(y) \mathrm{d} y .
$$

Naturally, $\sigma_{a(X, D)}(x, \xi)=a(x, \xi)$.

To contrast this with Euclidean (Hörmander's) symbol classes, we will write $b \in$ $S_{\rho, \delta}^{m}\left(\mathbb{R}^{n} \times \mathbb{R}^{n}\right)$ if $b \in C^{\infty}\left(\mathbb{R}^{n} \times \mathbb{R}^{n}\right)$ and if

$$
\left|\partial_{\xi}^{\alpha} \partial_{x}^{\beta} b(x, \xi)\right| \leq C_{b \alpha \beta m}\langle\xi\rangle^{m-\rho|\alpha|+\delta|\beta|}
$$

for every $x \in \mathbb{R}^{n}$, for every $\alpha, \beta \in \mathbb{N}_{0}^{n}$, and for all $\xi \in \mathbb{R}^{n}$. If in addition $b(\cdot, \xi)$ is $2 \pi$ periodic for every $\xi$ we will write $b \in S_{\rho, \delta}^{m}\left(\mathbb{T}^{n} \times \mathbb{R}^{n}\right)$. The corresponding (Euclidean) pseudo-differential operator is then given by

$$
b(X, D) f(x)=\int_{\mathbb{R}^{n}} \int_{\mathbb{T}^{n}} \mathrm{e}^{\mathrm{i}(x-y) \cdot \xi} b(x, \xi) f(y) \mathrm{d} y \mathrm{~d} \xi .
$$

The class $S_{\rho, \delta}^{m}\left(\mathbb{T}^{n} \times \mathbb{T}^{n} \times \mathbb{Z}^{n}\right)$ of toroidal compound symbols consists of the functions $a(x, y, \xi)$ which are smooth in $x$ and $y$ for all $\xi \in \mathbb{Z}^{n}$ and which satisfy

$$
\left|\triangle_{\xi}^{\alpha} \partial_{x}^{\beta} \partial_{y}^{\gamma} a(x, y, \xi)\right| \leq C_{a \alpha \beta \gamma m}\langle\xi\rangle^{m-\rho|\alpha|+\delta|\beta+\gamma|}
$$

for every $x, y \in \mathbb{T}^{n}$, for every $\alpha, \beta, \gamma \in \mathbb{N}_{0}^{n}$, and for all $\xi \in \mathbb{Z}^{n}$. Such a function $a$ will be also called a toroidal amplitude of order $m \in \mathbb{R}$ of type $(\rho, \delta)$. Formally we may also define

$$
(\mathrm{Op}(a) f)(x):=\sum_{\xi \in \mathbb{Z}^{n}} \int_{\mathbb{T}^{n}} \mathrm{e}^{\mathrm{i}(x-y) \cdot \xi} a(x, y, \xi) f(y) \mathrm{d} y
$$

for $f \in C^{\infty}\left(\mathbb{T}^{n}\right)$.

Remark 4.1. On $\mathbb{T}^{n}$, Hörmander's usual $(\rho, \delta)$ class of pseudo-differential operators $\operatorname{Op} S_{\rho, \delta}^{m}\left(\mathbb{R}^{n} \times \mathbb{R}^{n}\right)$ of order $m \in \mathbb{R}$ which are $2 \pi$-periodic in $x$ coincides with the class $\mathrm{Op} S_{\rho, \delta}^{m}\left(\mathbb{T}^{n} \times \mathbb{Z}^{n}\right)$, i.e.

$$
\mathrm{Op} S_{\rho, \delta}^{m}\left(\mathbb{T}^{n} \times \mathbb{R}^{n}\right)=\mathrm{Op} S_{\rho, \delta}^{m}\left(\mathbb{T}^{n} \times \mathbb{Z}^{n}\right)
$$

see e.g. [16], but in Theorem 5.2 we give a precise relation between actual symbols. The relation between the corresponding operators is then given in Theorem 6.4. 
Now we will discuss the calculus of pseudo-differential operators with toroidal symbols. For this, let us fix the notation first. We define

$$
D_{y}^{(\alpha)}=D_{y_{1}}^{\left(\alpha_{1}\right)} \cdots D_{y_{n}}^{\left(\alpha_{n}\right)}
$$

where $D_{y_{j}}^{(0)}=I$ and

$$
D_{y_{j}}^{(k+1)}=D_{y_{j}}^{(k)}\left(\frac{\partial}{\mathrm{i} \partial y_{j}}-k I\right)=\frac{\partial}{\mathrm{i} \partial y_{j}}\left(\frac{\partial}{\mathrm{i} \partial y_{j}}-I\right) \cdots\left(\frac{\partial}{\mathrm{i} \partial y_{j}}-k I\right) .
$$

Theorem 4.2 (Compound symbols). Let $0 \leq \delta<\rho \leq 1$. For every amplitude $a \in S_{\rho, \delta}^{m}\left(\mathbb{T}^{n} \times \mathbb{T}^{n} \times \mathbb{Z}^{n}\right)$ there exists a unique symbol $\sigma \in S_{\rho, \delta}^{m}\left(\mathbb{T}^{n} \times \mathbb{Z}^{n}\right)$ satisfying $\operatorname{Op}(a)=\operatorname{Op}(\sigma)$, where

$$
\left.\sigma(x, \xi) \sim \sum_{\alpha \geq 0} \frac{1}{\alpha !} \triangle_{\xi}^{\alpha} D_{y}^{(\alpha)} a(x, y, \xi)\right|_{y=x}
$$

The proof of this theorem is essentially the same as that of the one-dimensional case of $S_{1,0}^{m}(\mathbb{T} \times \mathbb{Z})$ analyzed in [32]. In [32] also the following results were proved for symbols in $S_{1,0}^{m}(\mathbb{T} \times \mathbb{Z}$ ) (see also [25]), and below we give their extensions to $(\rho, \delta)$ classes in $n$ dimensions. The possibility for such an extension comes from the discrete Taylor expansion and estimates for remainders in Theorem 3.4. We omit these lengthy proofs and refer to e.g. Theorem 8.2 for a more general version of this type of arguments.

Theorem 4.3 (Composition). Let $0 \leq \delta<\rho \leq 1$ and let $\sigma_{A} \in S_{\rho, \delta}^{m_{1}}\left(\mathbb{T}^{n} \times \mathbb{Z}^{n}\right)$ and $\sigma_{B} \in S_{\rho, \delta}^{m_{2}}\left(\mathbb{T}^{n} \times \mathbb{Z}^{n}\right)$. Then $\sigma_{A B} \in S_{\rho, \delta}^{m_{1}+m_{2}}\left(\mathbb{T}^{n} \times \mathbb{Z}^{n}\right)$ has the following asymptotic expansion:

$$
\sigma_{A B}(x, \xi) \sim \sum_{\alpha \geq 0} \frac{1}{\alpha !}\left(\triangle_{\xi}^{\alpha} \sigma_{A}(x, \xi)\right) D_{x}^{(\alpha)} \sigma_{B}(x, \xi)
$$

In particular, the symbol of the commutator $[A, B]=A B-B A$ belongs to the class $S_{\rho, \delta}^{m_{1}+m_{2}-1}\left(\mathbb{T}^{n} \times \mathbb{Z}^{n}\right)$.

Theorem 4.4 (Adjoint). Let $0 \leq \delta<\rho \leq 1$. Let $\sigma_{A} \in S_{\rho, \delta}^{m}\left(\mathbb{T}^{n} \times \mathbb{Z}^{n}\right)$. Then the symbol $\sigma_{A^{*}} \in S_{\rho, \delta}^{m}\left(\mathbb{T}^{n} \times \mathbb{Z}^{n}\right)$ of the adjoint operator $A^{*}$ has the asymptotic expansion

$$
\sigma_{A^{*}}(x, \xi) \sim \sum_{\alpha \geq 0} \frac{1}{\alpha !} \triangle_{\xi}^{\alpha} D_{x}^{(\alpha)} \overline{\sigma_{A}(x, \xi)} .
$$

From the asymptotic expansion for composition of pseudo-differential operators, we get an expansion for a parametrix of an elliptic operator:

Theorem 4.5 (Ellipticity). Let $\sigma_{A} \in S^{m}\left(\mathbb{T}^{n} \times \mathbb{Z}^{n}\right)$ be elliptic in the sense that there exist constants $C_{0}>0$ and $N_{0} \in \mathbb{N}_{0}$ such that

$$
\left|\sigma_{A}(x, \xi)\right| \geq C_{0}\langle\xi\rangle^{m}
$$

for all $(x, \xi) \in \mathbb{T}^{n} \times \mathbb{Z}^{n}$ for which $|\xi| \geq N_{0}$; this is equivalent to assuming that there exists $\sigma_{B} \in S^{-m}\left(\mathbb{T}^{n} \times \mathbb{Z}^{n}\right)$ such that $I-B A, I-A B$ are smoothing. Let $A \sim \sum_{j=0}^{\infty} A_{j}$, 
where $\sigma_{A_{j}} \in S^{m-j}\left(\mathbb{T}^{n} \times \mathbb{Z}^{n}\right)$. Then $B \sim \sum_{k=0}^{\infty} B_{k}$, where $B_{k} \in S^{-m-k}\left(\mathbb{T}^{n} \times \mathbb{Z}^{n}\right)$ is such that $\sigma_{B_{0}}(x, \xi)=1 / \sigma_{A_{0}}(x, \xi)$ for large enough $|\xi|$, and recursively

$$
\sigma_{B_{N}}(x, \xi)=\frac{-1}{\sigma_{A_{0}}(x, \xi)} \sum_{k=0}^{N-1} \sum_{j=0}^{N-k} \sum_{|\gamma|=N-j-k} \frac{1}{\gamma !}\left[\triangle_{\xi}^{\gamma} \sigma_{A_{j}}(x, \xi)\right] D_{x}^{(\gamma)} \sigma_{B_{k}}(x, \xi) .
$$

\section{EXTENSION OF TOROIDAL SYMBOLS}

It is often useful to extend toroidal symbols from $\mathbb{T}^{n} \times \mathbb{Z}^{n}$ to $\mathbb{T}^{n} \times \mathbb{R}^{n}$, ideally getting symbols in Hörmander's symbol classes. The case of $n=1$ and $(\rho, \delta)=(1,0)$ was considered in 32 and 25]. This extension can be done with a suitable convolution that respects the symbol inequalities. In the following, $\delta_{0, \xi}$ is the Kronecker delta at $0 \in \mathbb{Z}^{n}$, i.e. $\delta_{0,0}=1$, and $\delta_{0, \xi}=0$ if $\xi \neq 0$. First we prepare the following useful functions $\theta, \phi_{\alpha} \in \mathcal{S}\left(\mathbb{R}^{n}\right)$ :

Lemma 5.1. There exist functions $\phi_{\alpha} \in \mathcal{S}\left(\mathbb{R}^{n}\right)$ (for each $\alpha \in \mathbb{N}_{0}^{n}$ ) and a function $\theta \in \mathcal{S}\left(\mathbb{R}^{n}\right)$ such that

$$
\mathcal{P} \theta(x):=\sum_{k \in \mathbb{Z}^{n}} \theta(x+2 \pi k) \equiv 1,
$$

$\left.\left(\mathscr{F}_{\mathbb{R}^{n}} \theta\right)\right|_{\mathbb{Z}^{n}}(\xi)=\delta_{0, \xi}$ and $\partial_{\xi}^{\alpha}\left(\mathscr{F}_{\mathbb{R}^{n}} \theta\right)(\xi)=\bar{\triangle}_{\xi}^{\alpha} \phi_{\alpha}(\xi)$ for all $\xi \in \mathbb{Z}^{n}$.

Proof. Let us first consider the 1-dimensional case. Let $\theta=\theta_{1} \in C^{\infty}\left(\mathbb{R}^{1}\right)$ such that

$$
\operatorname{supp}\left(\theta_{1}\right) \subset(-2 \pi, 2 \pi), \quad \theta_{1}(-x)=\theta_{1}(x), \quad \theta_{1}(2 \pi-y)+\theta_{1}(y)=1
$$

for $x \in \mathbb{R}$ and for $0 \leq y \leq 2 \pi$; these assumptions for $\theta_{1}$ are enough for us, and of course the choice is not unique. In any case, $\theta_{1} \in \mathcal{S}\left(\mathbb{R}^{1}\right)$, so that also $\mathscr{F}_{\mathbb{R}} \theta_{1} \in \mathcal{S}\left(\mathbb{R}^{1}\right)$. If $\xi \in \mathbb{Z}^{1}$ then we have

$$
\mathscr{F}_{\mathbb{R}} \theta_{1}(\xi)=\int_{\mathbb{R}^{1}} \theta_{1}(x) \mathrm{e}^{-\mathrm{i} x \cdot \xi} \mathrm{d} x=\int_{0}^{2 \pi}\left(\theta_{1}(x-2 \pi)+\theta_{1}(x)\right) \mathrm{e}^{-\mathrm{i} x \cdot \xi} \mathrm{d} x=\delta_{0, \xi} .
$$

If a desired $\phi_{\alpha} \in \mathcal{S}\left(\mathbb{R}^{1}\right)$ exists, it must satisfy

$$
\int_{\mathbb{R}^{1}} \mathrm{e}^{\mathrm{i} x \cdot \xi} \partial_{\xi}^{\alpha}\left(\mathscr{F}_{\mathbb{R}} \theta_{1}\right)(\xi) \mathrm{d} \xi=\int_{\mathbb{R}^{1}} \mathrm{e}^{\mathrm{i} x \cdot \xi} \bar{\triangle}_{\xi}^{\alpha} \phi_{\alpha}(\xi) \mathrm{d} \xi=\left(1-\mathrm{e}^{\mathrm{i} x}\right)^{\alpha} \int_{\mathbb{R}^{1}} \mathrm{e}^{\mathrm{i} x \cdot \xi} \phi_{\alpha}(\xi) \mathrm{d} \xi
$$

due to the bijectivity of $\mathscr{F}_{\mathbb{R}}: \mathcal{S}\left(\mathbb{R}^{1}\right) \rightarrow \mathcal{S}\left(\mathbb{R}^{1}\right)$. Integration by parts leads to the formula

$$
(-\mathrm{i} x)^{\alpha} \theta_{1}(x)=\left(1-\mathrm{e}^{\mathrm{i} x}\right)^{\alpha}\left(\mathscr{F}_{\mathbb{R}}^{-1} \phi_{\alpha}\right)(x) .
$$

Thus

$$
\left(\mathscr{F}_{\mathbb{R}}^{-1} \phi_{\alpha}\right)(x)=\left\{\begin{aligned}
\left(\frac{-\mathrm{i} x}{1-\mathrm{e}^{\mathrm{i} x}}\right)^{\alpha} \theta_{1}(x), & \text { if } 0<|x|<2 \pi \\
1, & \text { if } x=0 \\
0, & \text { if }|x| \geq 2 \pi .
\end{aligned}\right.
$$

The general $n$-dimensional case is reduced to the 1-dimensional case, since mapping $\theta=\left(x \mapsto \theta_{1}\left(x_{1}\right) \theta_{1}\left(x_{2}\right) \cdots \theta_{1}\left(x_{n}\right)\right) \in \mathcal{S}\left(\mathbb{R}^{n}\right)$ has the desired properties.

Theorem 5.2. Let $0<\rho \leq 1$ and $0 \leq \delta \leq 1$. Symbol $\widetilde{a} \in S_{\rho, \delta}^{m}\left(\mathbb{T}^{n} \times \mathbb{Z}^{n}\right)$ is a toroidal symbol if and only if there exists an Euclidean symbol $a \in S_{\rho, \delta}^{m}\left(\mathbb{T}^{n} \times \mathbb{R}^{n}\right)$ such that $\widetilde{a}=\left.a\right|_{\mathbb{T}^{n} \times \mathbb{Z}^{n}}$. Moreover, this extended symbol $a$ is unique modulo $S^{-\infty}\left(\mathbb{T}^{n} \times \mathbb{R}^{n}\right)$. 
The relation between the corresponding pseudo-differential operators will be given in Theorem 6.4.

Proof. Let us first prove the "if" part. Let $a \in S_{\rho, \delta}^{m}\left(\mathbb{R}^{n} \times \mathbb{R}^{n}\right)$, and in this part we can actually allow any $\rho$ and $\delta$, for example $0 \leq \rho, \delta \leq 1$. By the Lagrange Mean Value Theorem, if $|\alpha|=1$ then

$$
\triangle_{\xi}^{\alpha} \partial_{x}^{\beta} \widetilde{a}(x, \xi)=\triangle_{\xi}^{\alpha} \partial_{x}^{\beta} a(x, \xi)=\left.\partial_{\xi}^{\alpha} \partial_{x}^{\beta} a(x, \xi)\right|_{\xi=\eta},
$$

where $\eta$ is on the line from $\xi$ to $\xi+\alpha$. For a general multi-index $\alpha \in \mathbb{N}_{0}^{n}$, we also have

$$
\triangle_{\xi}^{\alpha} \partial_{x}^{\beta} \widetilde{a}(x, \xi)=\left.\partial_{\xi}^{\alpha} \partial_{x}^{\beta} a(x, \xi)\right|_{\xi=\eta}
$$

for some $\eta \in Q:=\left[\xi_{1}, \xi_{1}+\alpha_{1}\right] \times \cdots \times\left[\xi_{n}, \xi_{n}+\alpha_{n}\right]$. This can be shown by induction. Indeed, let us write $\alpha=\delta+\gamma$ for some $\delta=e_{j}$. Then we can calculate

$$
\begin{aligned}
\triangle_{\xi}^{\alpha} \partial_{x}^{\beta} \widetilde{a}(x, \xi) & =\triangle_{\xi}^{\delta}\left(\triangle_{\xi}^{\gamma} \partial_{x}^{\beta} \widetilde{a}\right)(x, \xi)=\triangle_{\xi}^{e_{j}}\left(\left.\partial_{\xi}^{\gamma} \partial_{x}^{\beta} a(x, \xi)\right|_{\xi=\zeta}\right) \\
& =\partial_{\xi}^{\gamma} \partial_{x}^{\beta} a\left(x, \zeta+e_{j}\right)-\partial_{\xi}^{\gamma} \partial_{x}^{\beta} a(x, \zeta)=\left.\partial_{\xi}^{\alpha} \partial_{x}^{\beta} a(x, \xi)\right|_{\xi=\eta}
\end{aligned}
$$

for some $\zeta$ and $\eta$, where we used the induction hypothesis in the first line. Therefore, we can estimate

$$
\left|\triangle_{\xi}^{\alpha} \partial_{x}^{\beta} \widetilde{a}(x, \xi)\right|=\left|\partial_{\xi}^{\alpha} \partial_{x}^{\beta} a(x, \xi)\right|_{\xi=\eta \in Q} \mid \leq C_{\alpha \beta m}\langle\eta\rangle^{m-\rho|\alpha|+\delta|\beta|} \leq C_{\alpha \beta m}^{\prime}\langle\xi\rangle^{m-\rho|\alpha|+\delta|\beta|} .
$$

Let us now prove the "only if" part. First we show uniqueness. Let $a, b \in S_{\rho, \delta}^{m}\left(\mathbb{T}^{n} \times\right.$

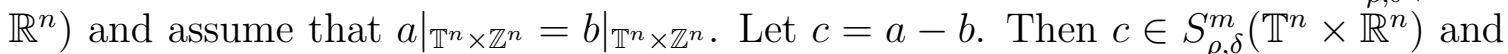
it satisfies $\left.c\right|_{\mathbb{T}^{n} \times \mathbb{Z}^{n}}=0$. If $\xi \in \mathbb{R}^{n} \backslash \mathbb{Z}^{n}$, choose $\eta \in \mathbb{Z}^{n}$ that is the nearest point (or one of the nearest points) to $\xi$. Then we have the first order Taylor expansion

$$
\begin{aligned}
c(x, \xi) & =c(x, \eta)+\sum_{\alpha:|\alpha|=1} r_{\alpha}(x, \xi, \xi-\eta)(\xi-\eta)^{\alpha} \\
& =\sum_{\alpha:|\alpha|=1} r_{\alpha}(x, \xi, \xi-\eta)(\xi-\eta)^{\alpha}
\end{aligned}
$$

where

$$
r_{\alpha}(x, \xi, \theta)=\int_{0}^{1}(1-t) \partial_{\xi}^{\alpha} c(x, \xi+t \theta) \mathrm{d} t .
$$

Hence we have $|c(x, \xi)| \leq C\langle\xi\rangle^{m-\rho}$. Continuing the argument inductively for $c$ and its derivatives, we obtain the uniqueness modulo $S^{-\infty}\left(\mathbb{T}^{n} \times \mathbb{R}^{n}\right)$.

Let us now show the existence. Let $\theta \in \mathcal{S}\left(\mathbb{R}^{n}\right)$ be as in the Lemma 5.1. Define $a: \mathbb{T}^{n} \times \mathbb{R}^{n} \rightarrow \mathbb{C}$ by

$$
a(x, \xi):=\sum_{\eta \in \mathbb{Z}^{n}}\left(\mathscr{F}_{\mathbb{R}^{n}} \theta\right)(\xi-\eta) \widetilde{a}(x, \eta) .
$$

It is easy to see that $\widetilde{a}=\left.a\right|_{\mathbb{T}^{n} \times \mathbb{Z}^{n}}$. Furthermore, we have

$$
\begin{aligned}
\left|\partial_{\xi}^{\alpha} \partial_{x}^{\beta} a(x, \xi)\right| & =\left|\sum_{\eta \in \mathbb{Z}^{n}} \partial_{\xi}^{\alpha}\left(\mathscr{F}_{\mathbb{R}^{n}} \theta\right)(\xi-\eta) \partial_{x}^{\beta} \widetilde{a}(x, \eta)\right| \\
& =\left|\sum_{\eta \in \mathbb{Z}^{n}} \bar{\triangle}_{\xi}^{\alpha} \phi_{\alpha}(\xi-\eta) \partial_{x}^{\beta} \widetilde{a}(x, \eta)\right|
\end{aligned}
$$




$$
\begin{aligned}
& =\left|\sum_{\eta \in \mathbb{Z}^{n}} \phi_{\alpha}(\xi-\eta) \triangle_{\eta}^{\alpha} \partial_{x}^{\beta} \widetilde{a}(x, \eta)(-1)^{|\alpha|}\right| \\
& \leq \sum_{\eta \in \mathbb{Z}^{n}}\left|\phi_{\alpha}(\xi-\eta)\right| C_{\alpha \beta m}\langle\eta\rangle^{m-\rho|\alpha|+\delta|\beta|} \\
& \leq C_{\alpha \beta m}^{\prime}\langle\xi\rangle^{m-\rho|\alpha|+\delta|\beta|} \sum_{\eta \in \mathbb{Z}^{n}}\left|\phi_{\alpha}(\eta)\right|\langle\eta\rangle^{|m-\rho| \alpha|+\delta| \beta||} \\
& \leq C_{\alpha \beta m}^{\prime \prime}\langle\xi\rangle^{m-\rho|\alpha|+\delta|\beta|},
\end{aligned}
$$

where we used the summation by parts formula (3.1). In the last two lines we also used that $\phi_{\alpha} \in \mathcal{S}\left(\mathbb{R}^{n}\right)$ and also a simple fact that for $p>0$ we have $\langle\xi+\eta\rangle^{p} \leq\langle\xi\rangle^{p}\langle\eta\rangle^{p}$ and $\langle\xi+\eta\rangle^{-p}\langle\eta\rangle^{-p} \leq\langle\xi\rangle^{-p}$, for all $\xi, \eta \in \mathbb{R}^{n}$. Thus $a \in S_{\rho, \delta}^{m}\left(\mathbb{T}^{n} \times \mathbb{R}^{n}\right)$.

\section{Periodization of PSEUdo-Differential operators}

In this section we describe the relation between operators with Euclidean and toroidal quantizations and between operators corresponding to symbols $a(x, \xi)$ and $\widetilde{a}=\left.a\right|_{\mathbb{T}^{n} \times \mathbb{Z}^{n}}$, given by the operator of periodization of functions.

First we state a property of a pseudo-differential operator $a(X, D)$ to map the space $\mathcal{S}\left(\mathbb{R}^{n}\right)$ into itself, which will be of importance. The following class will be sufficient for our purposes, and the proof is straightforward.

Proposition 6.1. Let $a=a(x, \xi) \in C^{\infty}\left(\mathbb{R}^{n} \times \mathbb{R}^{n}\right)$ and assume that there exist $\epsilon>0$ and $N \in \mathbb{R}$ such that for every $\alpha, \beta$ there are constants $C_{\alpha \beta}$ and $M(\alpha, \beta)$ such that the estimate

$$
\left|\partial_{x}^{\alpha} \partial_{\xi}^{\beta} a(x, \xi)\right| \leq C_{\alpha \beta}\langle x\rangle^{N+(1-\epsilon)|\beta|}\langle\xi\rangle^{M(\alpha, \beta)}
$$

holds for all $x, \xi \in \mathbb{R}^{n}$. Then pseudo-differential operator $a(X, D)$ with symbol $a(x, \xi)$ is continuous from $\mathcal{S}\left(\mathbb{R}^{n}\right)$ to $\mathcal{S}\left(\mathbb{R}^{n}\right)$.

Before analyzing the relation between operators with Euclidean and toroidal quantizations, we will describe the periodization operator that will be of importance for such analysis.

Theorem 6.2 (Periodization). The periodization $\mathcal{P} f: \mathbb{R}^{n} \rightarrow \mathbb{C}$ of a function $f \in$ $\mathcal{S}\left(\mathbb{R}^{n}\right)$ is defined by

$$
\mathcal{P} f(x):=\sum_{k \in \mathbb{Z}^{n}} f(x+2 \pi k) .
$$

Then $\mathcal{P}: \mathcal{S}\left(\mathbb{R}^{n}\right) \rightarrow C^{\infty}\left(\mathbb{T}^{n}\right)$ is surjective and $\|\mathcal{P} f\|_{L^{1}\left(\mathbb{T}^{n}\right)} \leq\|f\|_{L^{1}\left(\mathbb{R}^{n}\right)}$. Moreover, we have

$$
\mathcal{P} f(x)=\mathscr{F}_{\mathbb{T}^{n}}^{-1}\left(\left.\left(\mathscr{F}_{\mathbb{R}^{n}} f\right)\right|_{\mathbb{Z}^{n}}\right)(x)
$$

and

$$
\sum_{k \in \mathbb{Z}^{n}} f(x+2 \pi k)=\sum_{\xi \in \mathbb{Z}^{n}} \mathrm{e}^{\mathrm{i} x \cdot \xi}\left(\mathscr{F}_{\mathbb{R}^{n}} f\right)(\xi) .
$$

In particular, for $x=0$ this is the Poisson summation formula. 
We note that by Theorem 6.2 we may extend the periodization to $L^{1}\left(\mathbb{R}^{n}\right)$, and also this extension is surjective. This is actually rather trivial compared to the smooth case of Theorem 6.2 because we can find a preimage $f \in L^{1}\left(\mathbb{R}^{n}\right)$ of $g \in L^{1}\left(\mathbb{T}^{n}\right)$ under the periodization mapping $\mathcal{P}$ by for example setting $f=\left.g\right|_{[0,2 \pi]^{n}}$ and $f=0$ otherwise.

Proof. Let us show the surjectivity of $\mathcal{P}: \mathcal{S}\left(\mathbb{R}^{n}\right) \rightarrow C^{\infty}\left(\mathbb{T}^{n}\right)$. Let $\theta \in \mathcal{S}\left(\mathbb{R}^{n}\right)$ be a function defined in Lemma 5.1. Then for any $g \in C^{\infty}\left(\mathbb{T}^{n}\right)$ it holds that

$$
\mathcal{P}(g \theta)(x)=\sum_{k \in \mathbb{Z}^{n}} g(x+2 \pi k) \theta(x+2 \pi k)=g(x) \sum_{k \in \mathbb{Z}^{n}} \theta(x+2 \pi k)=g(x),
$$

where $g \theta$ is the product of $\theta$ with $2 \pi \mathbb{Z}^{n}$-periodic function $g$ on $\mathbb{R}^{n}$. We omit the straightforward proofs for the other claims.

We note that in the case of the $N$-inflated torus $N \mathbb{T}^{n}$ we can use the periodization operator $\mathcal{P}_{N}$ instead of $\mathcal{P}$, where $\mathcal{P}_{N}: \mathcal{S}\left(\mathbb{R}^{n}\right) \rightarrow C^{\infty}\left(N \mathbb{T}^{n}\right)$ can be defined by

$$
\left(\mathcal{P}_{N} f\right)(x)=\mathscr{F}_{N \mathbb{T}^{n}}^{-1}\left(\left.\mathscr{F}_{\mathbb{R}^{n}} f\right|_{\frac{1}{N} \mathbb{Z}^{n}}\right)(x), \quad x \in N \mathbb{T}^{n} .
$$

Let us now establish some basic properties of pseudo-differential operators with respect to periodization.

Definition 6.3. We will say that a function $a: \mathbb{R}^{n} \times \mathbb{R}^{n} \rightarrow \mathbb{C}$ is $2 \pi$-periodic (we will always mean that it is periodic with respect to the first variable $x \in \mathbb{R}^{n}$ ) if the function $x \mapsto a(x, \xi)$ is $2 \pi \mathbb{Z}^{n}$-periodic for all $\xi$.

As before, we use tilde to denote the restriction of $a \in C^{\infty}\left(\mathbb{R}^{n} \times \mathbb{R}^{n}\right)$ to $\mathbb{R}^{n} \times \mathbb{Z}^{n}$. If $a(x, \xi)$ is $2 \pi$-periodic, we can view it as a function on $\mathbb{T}^{n} \times \mathbb{Z}^{n}$, and we write $\widetilde{a}=\left.a\right|_{\mathbb{T}^{n} \times \mathbb{Z}^{n}}$.

Theorem 6.4. Let $a=a(x, \xi) \in C^{\infty}\left(\mathbb{R}^{n} \times \mathbb{R}^{n}\right)$ be $2 \pi$-periodic with respect to $x$ for every $\xi \in \mathbb{R}^{n}$. Assume that for every $\alpha, \beta \in \mathbb{N}_{0}^{n}$ there are constants $C_{\alpha \beta}$ and $M(\alpha, \beta)$ such that estimate

$$
\left|\partial_{x}^{\alpha} \partial_{\xi}^{\beta} a(x, \xi)\right| \leq C_{\alpha \beta}\langle\xi\rangle^{M(\alpha, \beta)}
$$

holds for all $x, \xi \in \mathbb{R}^{n}$. Let $\widetilde{a}=\left.a\right|_{\mathbb{T}^{n} \times \mathbb{Z}^{n}}$. Then

$$
\mathcal{P} \circ a(X, D) f=\widetilde{a}(X, D) \circ \mathcal{P} f
$$

for all $f \in \mathcal{S}\left(\mathbb{R}^{n}\right)$.

Note that it is not important in this theorem that $a$ is in a symbol class.

Remark 6.5. Note that by Proposition 6.1 both sides of (6.5) are well-defined functions in $C^{\infty}\left(\mathbb{T}^{n}\right)$. Moreover, equality (6.5) can be justified for $f$ in larger classes of functions. For example, (6.5) remains true if $f \in C_{0}^{k}\left(\mathbb{R}^{n}\right)$ is a $C^{k}$ compactly supported function for $k$ sufficiently large.

Proof of Theorem 6.4. Let $f \in \mathcal{S}\left(\mathbb{R}^{n}\right)$. Then we have

$$
\begin{aligned}
\mathcal{P}(a(X, D) f)(x) & =\sum_{k \in \mathbb{Z}^{n}}(a(X, D) f)(x+2 \pi k) \\
& =\sum_{k \in \mathbb{Z}^{n}} \int_{\mathbb{R}^{n}} \mathrm{e}^{\mathrm{i}(x+2 \pi k) \cdot \xi} a(x+2 \pi k, \xi)\left(\mathscr{F}_{\mathbb{R}^{n}} f\right)(\xi) \mathrm{d} \xi
\end{aligned}
$$




$$
\begin{aligned}
& =\int_{\mathbb{R}^{n}}\left(\sum_{k \in \mathbb{Z}^{n}} \mathrm{e}^{\mathrm{i} 2 \pi k \cdot \xi}\right) \mathrm{e}^{\mathrm{i} x \cdot \xi} a(x, \xi)\left(\mathscr{F}_{\mathbb{R}^{n}} f\right)(\xi) \mathrm{d} \xi \\
& =\int_{\mathbb{R}^{n}} \delta_{\mathbb{Z}^{n}}(\xi) \mathrm{e}^{\mathrm{i} x \cdot \xi} a(x, \xi)\left(\mathscr{F}_{\mathbb{R}^{n}} f\right)(\xi) \mathrm{d} \xi \\
& =\sum_{\xi \in \mathbb{Z}^{n}} \mathrm{e}^{\mathrm{i} x \cdot \xi} a(x, \xi)\left(\mathscr{F}_{\mathbb{R}^{n}} f\right)(\xi) \\
& =\sum_{\xi \in \mathbb{Z}^{n}} \mathrm{e}^{\mathrm{i} x \cdot \xi} a(x, \xi) \mathscr{F}_{\mathbb{T}^{n}}(\mathcal{P} f)(\xi) \\
& =\widetilde{a}(X, D)(\mathcal{P} f)(x) .
\end{aligned}
$$

As usual, these calculations can be justified in the sense of distributions.

Let us now formulate a useful corollary of Theorem 6.4 that will be of importance later, in particular in the proof of Theorem 8.4. If in Theorem 6.4 we take function $f$ such that $f=\left.g\right|_{[0,2 \pi]^{n}}$ for some $g \in C^{\infty}\left(\mathbb{T}^{n}\right)$, and $f=0$ otherwise, it follows immediately that $g=\mathcal{P} f$. Adjusting this argument by shifting the cube $[0,2 \pi]^{n}$ if necessary, we obtain

Corollary 6.6. Let $a=a(x, \xi)$ be as in Theorem 6.4. let $g \in C^{\infty}\left(\mathbb{T}^{n}\right)$, and let $V$ be an open cube in $\mathbb{R}^{n}$ with side length equal to $2 \pi$. Assume that the support of $\left.g\right|_{\bar{V}}$ is contained in $V$. Then we have the equality

$$
\widetilde{a}(X, D) g=\mathcal{P} \circ a(X, D)\left(\left.g\right|_{V}\right),
$$

where $\left.g\right|_{V}: \mathbb{R}^{n} \rightarrow \mathbb{C}$ is defined as the restriction of $g$ to $V$ and equal to zero outside of $V$.

Since we do not always have periodic symbols on $\mathbb{R}^{n}$ it may be convenient to periodize them. If $a(X, D)$ is a pseudo-differential operator with symbol $a(x, \xi)$, by $(\mathcal{P} a)(X, D)$ we will denote the pseudo-differential operator with symbol

$$
(\mathcal{P} a)(x, \xi)=\sum_{k \in \mathbb{Z}^{n}} a(x+2 \pi k, \xi) .
$$

This procedure makes sense if, for example, $a$ is in $L^{1}\left(\mathbb{R}^{n}\right)$ with respect to $x$.

In the following proposition we will assume that supports of symbols and functions are contained in $[-\pi, \pi]^{n}$. We note that this is not restrictive if these functions are already compactly supported. Indeed, if supports of $a(\cdot, \xi)$ and $f$ are contained in some compact set independent of $\xi$, we can find some $N \in \mathbb{N}$ such that they are contained in $[-N \pi, N \pi]^{n}$, and then use the analysis on the $N$-inflated torus, with periodization operator $\mathcal{P}_{N}$ instead of $\mathcal{P}$, defined in (6.4).

Proposition 6.7. Let $a=a(x, \xi)$ satisfy $\operatorname{supp} a \subset[-\pi, \pi]^{n} \times \mathbb{R}^{n}$ and be such that for every $\alpha, \beta \in \mathbb{N}_{0}^{n}$ there are constants $C_{\alpha \beta}$ and $M(\alpha, \beta)$ such that the estimate

$$
\left|\partial_{x}^{\alpha} \partial_{\xi}^{\beta} a(x, \xi)\right| \leq C_{\alpha \beta}\langle\xi\rangle^{M(\alpha, \beta)}
$$

holds for all $x, \xi \in \mathbb{R}^{n}$. Then we have

$$
a(X, D) f=(\mathcal{P} a)(X, D) f+R f
$$


for all $f \in C^{\infty}\left(\mathbb{R}^{n}\right)$ with supp $f \subset[-\pi, \pi]^{n}$. Operator $R$ extends to a smoothing pseudo-differential operator $R: \mathcal{D}^{\prime}\left(\mathbb{R}^{n}\right) \rightarrow \mathcal{S}\left(\mathbb{R}^{n}\right)$.

Proof. By the definition we can write

$$
(\mathcal{P} a)(X, D) f(x)=\sum_{k \in \mathbb{Z}^{n}} \int_{\mathbb{R}^{n}} \mathrm{e}^{\mathrm{i} x \cdot \xi} a(x+2 \pi k, \xi) \mathscr{F}_{\mathbb{R}^{n}} f(\xi) \mathrm{d} \xi,
$$

and let us define $R f:=a(X, D) f-(\mathcal{P} a)(X, D) f$. The assumption on the support of $a$ implies that for every $x$ there is only one $k \in \mathbb{Z}^{n}$ for which $a(x+2 \pi k, \xi) \neq 0$, so the sum consists of only one term. It follows that $R f(x)=0$ for $x \in[-\pi, \pi]^{n}$, because for such $x$ the non-zero term corresponds to $k=0$. Let now $x \in \mathbb{R}^{n} \backslash[-\pi, \pi]^{n}$. Since

$$
R f(x)=-\sum_{k \in \mathbb{Z}^{n}, k \neq 0} \int_{\mathbb{R}^{n}} \int_{\mathbb{R}^{n}} \mathrm{e}^{\mathrm{i}(x-y) \cdot \xi} a(x+2 \pi k, \xi) f(y) \mathrm{d} y \mathrm{~d} \xi
$$

is just a single term and $|x-y|>0$ on supp $f$, we can integrate by parts with respect to $\xi$ any number of times. This implies that $R \in \Psi^{-\infty}$ and that $R f$ decays at infinity faster than any power. The proof is complete since the same argument can be applied to the derivatives of $R f$.

Proposition 6.7 allows us to extend formula of Theorem 6.4 to compact perturbations of periodic symbols. We will use it when $a(X, D)$ is a sum of a constant coefficient operator and an operator with compactly (in $x$ ) supported symbol.

Corollary 6.8. Let $a(X, D)$ be an operator with symbol

$$
a(x, \xi)=a_{1}(x, \xi)+a_{0}(x, \xi),
$$

where $a_{1}$ is as in Theorem 6.4, $a_{1}$ is $2 \pi$-periodic in $x$ for every $\xi \in \mathbb{R}^{n}$, and $a_{0}$ is as in Proposition 6.7, supported in $[-\pi, \pi]^{n} \times \mathbb{R}^{n}$. Define

$$
\widetilde{b}(x, \xi):=\widetilde{a_{1}}(x, \xi)+\widetilde{\mathcal{P} a_{0}}(x, \xi), \quad x \in \mathbb{T}^{n}, \xi \in \mathbb{Z}^{n} .
$$

Then we have

$$
\mathcal{P} \circ a(X, D) f=\widetilde{b}(X, D) \circ \mathcal{P} f+\mathcal{P} \circ R f
$$

for all $f \in \mathcal{S}\left(\mathbb{R}^{n}\right)$, and operator $R$ extends to $R: \mathcal{D}^{\prime}\left(\mathbb{R}^{n}\right) \rightarrow \mathcal{S}\left(\mathbb{R}^{n}\right)$, so that $\mathcal{P} \circ R$ : $\mathcal{D}^{\prime}\left(\mathbb{R}^{n}\right) \rightarrow C^{\infty}\left(\mathbb{T}^{n}\right)$. Moreover, if $a_{1}, a_{0} \in S_{\rho, \delta}^{m}\left(\mathbb{R}^{n} \times \mathbb{R}^{n}\right)$, then $\widetilde{b} \in S_{\rho, \delta}^{m}\left(\mathbb{T}^{n} \times \mathbb{Z}^{n}\right)$.

Remark 6.9. Recalling Remark 6.5, (6.6) can be justified for larger function classes, e.g. for $f \in C_{0}^{k}\left(\mathbb{R}^{n}\right)$ for $k$ sufficiently large (which will be of use in Section 7 ).

Proof of Corollary 6.8. By Proposition 6.7 we can write

$$
a(X, D)=a_{1}(X, D)+\left(\mathcal{P} a_{0}\right)(X, D)+R,
$$

with $R: \mathcal{D}^{\prime}\left(\mathbb{R}^{n}\right) \rightarrow \mathcal{S}\left(\mathbb{R}^{n}\right)$. Let us define $b(x, \xi)=a_{1}(x, \xi)+\left(\mathcal{P} a_{0}\right)(x, \xi)$, so that $\widetilde{b}=\left.b\right|_{\mathbb{T}^{n} \times \mathbb{Z}^{n}}$. The symbol $b$ is $2 \pi$-periodic, hence for the operator $b(X, D)=a_{1}(X, D)+$ $\left(\mathcal{P} a_{0}\right)(X, D)$ by Theorem 6.4 we have

$$
\mathcal{P} \circ b(X, D)=\widetilde{b}(X, D) \circ \mathcal{P}=\widetilde{a_{1}}(X, D) \circ \mathcal{P}+\widetilde{\mathcal{P} a_{0}}(X, D) \circ \mathcal{P}
$$

Since $R: \mathcal{D}^{\prime}\left(\mathbb{R}^{n}\right) \rightarrow \mathcal{S}\left(\mathbb{R}^{n}\right)$, we also have $\mathcal{P} \circ R: \mathcal{D}^{\prime}\left(\mathbb{R}^{n}\right) \rightarrow C^{\infty}\left(\mathbb{T}^{n}\right)$. Finally, if $a_{1}, a_{0} \in S_{\rho, \delta}^{m}\left(\mathbb{R}^{n} \times \mathbb{R}^{n}\right)$, then $\tilde{b} \in S_{\rho, \delta}^{m}\left(\mathbb{T}^{n} \times \mathbb{Z}^{n}\right)$ by Theorem 5.2. The proof is complete. 


\section{TOROIDAL WAVE FRONT SETS}

Here we shall briefly study microlocal analysis not on the cotangent bundle of the torus but on $\mathbb{T}^{n} \times \mathbb{Z}^{n}$, which is better suited for Fourier series representations. Let us define mappings

$$
\begin{aligned}
\pi_{\mathbb{R}^{n}}: \mathbb{R}^{n} \backslash\{0\} \rightarrow \mathbb{S}^{n-1}, & \pi_{\mathbb{R}^{n}}(\xi)=\xi /|\xi|, \\
\pi_{\mathbb{T}^{n} \times \mathbb{R}^{n}}: \mathbb{T}^{n} \times\left(\mathbb{R}^{n} \backslash\{0\}\right) \rightarrow \mathbb{T}^{n} \times \mathbb{S}^{n-1}, & \pi_{\mathbb{T}^{n} \times \mathbb{R}^{n}}(x, \xi)=(x, \xi /|\xi|) .
\end{aligned}
$$

We say that $K \subset \mathbb{R}^{n} \backslash\{0\}$ is a cone in $\mathbb{R}^{n}$ if $\xi \in K$ and $\lambda>0$ imply $\lambda \xi \in K$. We say that $\Gamma \subset \mathbb{Z}^{n} \backslash\{0\}$ is a discrete cone if $\Gamma=\mathbb{Z}^{n} \cap K$ for some cone $K$ in $\mathbb{R}^{n}$; moreover, if here $K$ is open then $\Gamma$ is called and open discrete cone. We omit the straightforward proof of the following result:

Proposition 7.1. $\Gamma \subset \mathbb{Z}^{n} \backslash\{0\}$ is a discrete cone if and only if $\Gamma=\mathbb{Z}^{n} \cap \pi_{\mathbb{R}^{n}}^{-1}\left(\pi_{\mathbb{R}^{n}}(\Gamma)\right)$.

Let $u \in \mathcal{D}^{\prime}\left(\mathbb{T}^{n}\right)$. The toroidal wave front set $\mathrm{WF}_{T}(u) \subset \mathbb{T}^{n} \times\left(\mathbb{Z}^{n} \backslash\{0\}\right)$ is defined as follows: we say that $\left(x_{0}, \xi_{0}\right) \in \mathbb{T}^{n} \times\left(\mathbb{Z}^{n} \backslash\{0\}\right)$ does not belong to $\mathrm{WF}_{T}(u)$ if and only if there exist $\chi \in C^{\infty}\left(\mathbb{T}^{n}\right)$ and an open discrete cone $\Gamma \subset \mathbb{Z}^{n} \backslash\{0\}$ such that $\chi\left(x_{0}\right) \neq 0, \xi_{0} \in \Gamma$ and

$$
\forall N>0 \exists C_{N}<\infty \forall \xi \in \Gamma:\left|\mathscr{F}_{\mathbb{T}^{n}}(\chi u)(\xi)\right| \leq C_{N}\langle\xi\rangle^{-N} ;
$$

in such a case we say that $\mathscr{F}_{\mathbb{T}^{n}}(\chi u)$ decays rapidly in $\Gamma$.

We say that a pseudo-differential operator $A \in \Psi^{m}\left(\mathbb{T}^{n} \times \mathbb{Z}^{n}\right)=\mathrm{Op} S^{m}\left(\mathbb{T}^{n} \times \mathbb{Z}^{n}\right)$ is elliptic at the point $\left(x_{0}, \xi_{0}\right) \in \mathbb{T}^{n} \times\left(\mathbb{Z}^{n} \backslash\{0\}\right)$ if its toroidal symbol $\sigma_{A}: \mathbb{T}^{n} \times \mathbb{Z}^{n} \rightarrow \mathbb{C}$ satisfies

$$
\left|\sigma_{A}\left(x_{0}, \xi\right)\right| \geq C\langle\xi\rangle^{m}
$$

for some constant $C>0$ as $|\xi| \rightarrow \infty$, where $\xi \in \Gamma$ and $\Gamma \subset \mathbb{Z}^{n} \backslash\{0\}$ is an open discrete cone containing $\xi_{0}$. Should $\xi \mapsto \sigma_{A}\left(x_{0}, \xi\right)$ be rapidly decaying in an open discrete cone containing $\xi_{0}$ then $A$ is said to be smoothing at $\left(x_{0}, \xi_{0}\right)$. The toroidal characteristic set of $A \in \Psi^{m}\left(\mathbb{T}^{n} \times \mathbb{Z}^{n}\right)$ is

$$
\operatorname{char}_{T}(A):=\left\{\left(x_{0}, \xi_{0}\right) \in \mathbb{T}^{n} \times\left(\mathbb{Z}^{n} \backslash\{0\}\right): A \text { is not elliptic at }\left(x_{0}, \xi_{0}\right)\right\},
$$

and the toroidal wave front set of $A$ is

$$
\mathrm{WF}_{T}(A):=\left\{\left(x_{0}, \xi_{0}\right) \in \mathbb{T}^{n} \times\left(\mathbb{Z}^{n} \backslash\{0\}\right): A \text { is not smoothing at }\left(x_{0}, \xi_{0}\right)\right\} .
$$

Now,

$$
\mathrm{WF}_{T}(A) \cup \operatorname{char}_{T}(A)=\mathbb{T}^{n} \times\left(\mathbb{Z}^{n} \backslash\{0\}\right)
$$

because if $(x, \xi) \notin \operatorname{char}_{T}(A)$, it means that $A$ is elliptic at $(x, \xi)$, and hence not smoothing. It can be shown that $\mathrm{WF}_{T}(A)=\emptyset$ if and only if $A$ is smoothing, i.e. maps $\mathcal{D}^{\prime}\left(\mathbb{T}^{n}\right)$ to $C^{\infty}\left(\mathbb{T}^{n}\right)$ (equivalently, the Schwartz kernel is $C^{\infty}$-smooth).

Proposition 7.2. Let $A, B \in \mathrm{Op} S^{m}\left(\mathbb{T}^{n} \times \mathbb{Z}^{n}\right)$. Then

$$
\mathrm{WF}_{T}(A B) \subset \mathrm{WF}_{T}(A) \cap \mathrm{WF}_{T}(B) .
$$

Proof. By Theorem 4.3 applied to pseudo-differential operators $A$ and $B$ we notice that the toroidal symbol of $A B \in \mathrm{Op} S^{2 m}\left(\mathbb{T}^{n} \times \mathbb{Z}^{n}\right)$ has an asymptotic expansion

$$
\sigma_{A B}(x, \xi) \sim \sum_{\alpha \geq 0} \frac{1}{\alpha !}\left(\triangle_{\xi}^{\alpha} \sigma_{A}(x, \xi)\right) D_{x}^{(\alpha)} \sigma_{B}(x, \xi)
$$




$$
\sim \sum_{\alpha \geq 0} \frac{1}{\alpha !}\left(\partial_{\xi}^{\alpha} \sigma_{A}(x, \xi)\right) \partial_{x}^{\alpha} \sigma_{B}(x, \xi)
$$

where in the latter expansion we have used smooth extensions of toroidal symbols. This expansion tells that $A B$ is smoothing at $\left(x_{0}, \xi_{0}\right)$ if $A$ or $B$ is smoothing at $\left(x_{0}, \xi_{0}\right)$.

The notion of the toroidal wave front set is compatible with the action of pseudodifferential operators:

Proposition 7.3. Let $u \in \mathcal{D}^{\prime}\left(\mathbb{T}^{n}\right)$ and $A \in \mathrm{Op} S_{\rho, \delta}^{m}\left(\mathbb{T}^{n} \times \mathbb{Z}^{n}\right)$, where $0 \leq \rho \leq 1$, $0 \leq \delta<1$. Then

$$
\mathrm{WF}_{T}(A u) \subset \mathrm{WF}_{T}(u)
$$

Especially, if $\varphi \in C^{\infty}\left(\mathbb{T}^{n}\right)$ does not vanish, then $\mathrm{WF}_{T}(\varphi u)=\mathrm{WF}_{T}(u)$.

We omit the proof which is straightforward.

We will not pursue the complete analysis of toroidal wave front sets here because most of their properties can be obtained from the known properties of the usual wave front sets and the following relation, where WF $(u)$ stands for the usual Hörmander's wave front set of a distribution $u$.

Theorem 7.4. Let $u \in \mathcal{D}^{\prime}\left(\mathbb{T}^{n}\right)$. Then

$$
\mathrm{WF}_{T}(u)=\left(\mathbb{T}^{n} \times \mathbb{Z}^{n}\right) \cap \mathrm{WF}(u) .
$$

Proof. Without loss of generality, let $u \in C^{k}\left(\mathbb{T}^{n}\right)$ for some large $k$, and let $\chi \in$ $C_{0}^{\infty}\left(\mathbb{R}^{n}\right)$ such that $\operatorname{supp}(\chi) \subset(0,2 \pi)^{n}$. If $\mathscr{F}_{\mathbb{R}^{n}}(\chi u)$ decays rapidly in an open cone $K \subset \mathbb{R}^{n}$ then $\mathscr{F}_{\mathbb{T}^{n}}(\mathcal{P}(\chi u))=\left.\mathscr{F}_{\mathbb{R}^{n}}(\chi u)\right|_{\mathbb{Z}^{n}}$ decays rapidly in the open discrete cone $\mathbb{Z}^{n} \cap K$. Hence $\mathrm{WF}_{T}(u) \subset\left(\mathbb{T}^{n} \times \mathbb{Z}^{n}\right) \cap \mathrm{WF}(u)$.

Next, we need to show that $\left(\mathbb{T}^{n} \times \mathbb{Z}^{n}\right) \backslash \mathrm{WF}_{T}(u) \subset\left(\mathbb{T}^{n} \times \mathbb{Z}^{n}\right) \backslash \mathrm{WF}(u)$. Let $\left(x_{0}, \xi_{0}\right) \in\left(\mathbb{T}^{n} \times \mathbb{Z}^{n}\right) \backslash \mathrm{WF}_{T}(u)$ (where $\left.\xi_{0} \neq 0\right)$. We must show that $\left(x_{0}, \xi_{0}\right) \notin \mathrm{WF}(u)$. There exist $\chi \in C^{\infty}\left(\mathbb{T}^{n}\right)$ (we may assume that $\operatorname{supp}(\chi) \subset(0,2 \pi)^{n}$ as above) and an open cone $K \subset \mathbb{R}^{n}$ such that $\chi\left(x_{0}\right) \neq 0, \xi_{0} \in \mathbb{Z}^{n} \cap K$ and that $\mathscr{F}_{\mathbb{T}^{n}}(\mathcal{P}(\chi u))$ decays rapidly in $\mathbb{Z}^{n} \cap K$.

Let $K_{1} \subset \mathbb{R}^{n}$ be an open cone such that $\xi_{0} \in K_{1} \subset K$ and that the closure $\overline{K_{1}} \subset K \cup\{0\}$. Take any function $w \in C^{\infty}\left(\mathbb{S}^{n-1}\right)$ such that

$$
w(\omega)= \begin{cases}1, & \text { if } \omega \in \mathbb{S}^{n-1} \cap K_{1}, \\ 0, & \text { if } \omega \in \mathbb{S}^{n-1} \backslash K .\end{cases}
$$

Let $a \in C^{\infty}\left(\mathbb{R}^{n} \times \mathbb{R}^{n}\right)$ be independent of $x$ and such that $a(x, \xi)=w(\xi /|\xi|)$ whenever $|\xi| \geq 1$. Then $a \in S^{0}\left(\mathbb{R}^{n} \times \mathbb{R}^{n}\right)$. Let $\widetilde{a}=\left.a\right|_{\mathbb{T}^{n} \times \mathbb{Z}^{n}}$, so that $\widetilde{a} \in S^{0}\left(\mathbb{T}^{n} \times \mathbb{Z}^{n}\right)$ by Theorem 5.2 .

By Corollary 6.8, we have $\mathcal{P}(\chi a(X, D) f)=\mathcal{P}(\chi) \widetilde{a}(X, D)(\mathcal{P} f)+\mathcal{P}(R f)$ for all Schwartz test functions $f$, for a smoothing operator $R: \mathcal{D}^{\prime}\left(\mathbb{R}^{n}\right) \rightarrow \mathcal{S}\left(\mathbb{R}^{n}\right)$. By Remark 6.9 we also have $\mathcal{P}(\chi a(X, D)(\chi u))=\mathcal{P}(\chi) \widetilde{a}(X, D)(\mathcal{P}(\chi u))+\mathcal{P}(R(\chi u))$, where the right hand side belongs to $C^{\infty}\left(\mathbb{T}^{n}\right)$, since its Fourier coefficients decay rapidly on the whole $\mathbb{Z}^{n}$. Therefore also $\mathcal{P}(\chi a(X, D)(\chi u))$ belongs to $C^{\infty}\left(\mathbb{T}^{n}\right)$.

Thus $\chi a(X, D)(\chi u) \in C_{0}^{\infty}\left(\mathbb{R}^{n}\right)$. Let $\xi \in K_{1}$ such that $|\xi| \geq 1$. Then we have $\mathscr{F}_{\mathbb{R}^{n}}(a(X, D)(\chi u))(\xi)=w(\xi /|\xi|) \mathscr{F}_{\mathbb{R}^{n}}(\chi u)(\xi)=\mathscr{F}_{\mathbb{R}^{n}}(\chi u)(\xi)$. Thus $\mathscr{F}_{\mathbb{R}^{n}}(\chi u)$ decays rapidly on $K_{1}$. Therefore $\left(x_{0}, \xi_{0}\right)$ does not belong to $\operatorname{WF}(u)$. 


\section{Calculus of Fourier series operators}

In this section we consider analogues of Fourier integral operators on the torus $\mathbb{T}^{n}$. We will call such operators Fourier series operators and study their composition formulae with pseudo-differential operators on the torus. Fourier series operators will be defined by the formula

$$
T u(x):=\sum_{\xi \in \mathbb{Z}^{n}} \int_{\mathbb{T}^{n}} \mathrm{e}^{\mathrm{i}(\phi(x, \xi)-y \cdot \xi)} a(x, y, \xi) u(y) \mathrm{d} y,
$$

where $a \in C^{\infty}\left(\mathbb{T}^{n} \times \mathbb{T}^{n} \times \mathbb{Z}^{n}\right)$ is a toroidal amplitude and $\phi$ is a real-valued phase function. We note that if $u \in C^{\infty}\left(\mathbb{T}^{n}\right)$, for function $T u$ to be well-defined on the torus we need that the integral (8.1) is $2 \pi$-periodic in $x$. Therefore, by identifying functions on $\mathbb{T}^{n}$ with $2 \pi$-periodic functions on $\mathbb{R}^{n}$, we will require that the phase function $\phi: \mathbb{R}^{n} \times \mathbb{Z}^{n} \rightarrow \mathbb{R}$ is such that function $x \mapsto \mathrm{e}^{\mathrm{i} \phi(x, \xi)}$ is $2 \pi$-periodic for all $\xi \in \mathbb{Z}^{n}$. Then operator $T: C^{\infty}\left(\mathbb{T}^{n}\right) \rightarrow \mathcal{D}^{\prime}\left(\mathbb{T}^{n}\right)$ can be justified in the usual way for oscillatory integrals.

Remark 8.1. Assume that function $\phi: \mathbb{R}^{n} \times \mathbb{Z}^{n} \rightarrow \mathbb{R}$ is in $C^{k}$ with respect to $x$ for all $\xi \in \mathbb{Z}^{n}$. Assume also that function $x \mapsto \mathrm{e}^{\mathrm{i} \phi(x, \xi)}$ is $2 \pi$-periodic for all $\xi \in \mathbb{Z}^{n}$. Then functions $x \mapsto \partial_{x}^{\alpha} \phi(x, \xi)$ are $2 \pi$-periodic for all $\xi \in \mathbb{Z}^{n}$ and all $\alpha \in \mathbb{N}_{0}^{n}$ with $1 \leq|\alpha| \leq k$.

Composition formulae of this section can be compared with those obtained by Ruzhansky and Sugimoto [19, 21], but the assumptions on the regularity or boundedness of higher order derivatives of phases and amplitudes are redundant here thanks to the fact that $\xi \in \mathbb{Z}^{n}$ takes only discrete values.

We recall the notation for the toroidal version of Taylor polynomials and the corresponding derivatives introduced in (3.2) and (4.3), which will be used in the formulation of the following theorems. We also define

$$
\left(-D_{y}\right)^{(\alpha)}=\left(-D_{y_{1}}\right)^{\left(\alpha_{1}\right)} \cdots\left(-D_{y_{n}}\right)^{\left(\alpha_{n}\right)}
$$

where $-D_{y_{j}}^{(0)}=I$ and

$$
\left(-D_{y_{j}}\right)^{(k+1)}=\left(-D_{y_{j}}\right)^{(k)}\left(-\frac{\partial}{\mathrm{i} \partial y_{j}}-k I\right)=-\frac{\partial}{\mathrm{i} \partial y_{j}}\left(-\frac{\partial}{\mathrm{i} \partial y_{j}}-I\right) \cdots\left(-\frac{\partial}{\mathrm{i} \partial y_{j}}-k I\right) .
$$

We also recall definition (4.1) of pseudo-differential operators with toroidal symbols.

Theorem 8.2 (Composition $T \circ P(X, D)$ ). Let $\phi: \mathbb{R}^{n} \times \mathbb{Z}^{n} \rightarrow \mathbb{R}$ be such that function $x \mapsto \mathrm{e}^{\mathrm{i} \phi(x, \xi)}$ is $2 \pi$-periodic for all $\xi \in \mathbb{Z}^{n}$. Let $T: C^{\infty}\left(\mathbb{T}^{n}\right) \rightarrow \mathcal{D}^{\prime}\left(\mathbb{T}^{n}\right)$ be defined by

$$
T u(x):=\sum_{\xi \in \mathbb{Z}^{n}} \int_{\mathbb{T}^{n}} \mathrm{e}^{\mathrm{i}(\phi(x, \xi)-y \cdot \xi)} a(x, y, \xi) u(y) \mathrm{d} y,
$$

where the toroidal amplitude $a \in C^{\infty}\left(\mathbb{T}^{n} \times \mathbb{T}^{n} \times \mathbb{Z}^{n}\right)$ satisfies

$$
\left|\partial_{x}^{\alpha} \partial_{y}^{\beta} a(x, y, \xi)\right| \leq C_{\alpha \beta m}\langle\xi\rangle^{m}
$$

for every $x, y \in \mathbb{T}^{n}, \xi \in \mathbb{Z}^{n}$ and $\alpha, \beta \in \mathbb{N}_{0}^{n}$. Let $p \in S^{\ell}\left(\mathbb{T}^{n} \times \mathbb{Z}^{n}\right)$ be a toroidal symbol and $P=p(X, D)$ the corresponding pseudo-differential operator. Then the 
composition TP has the form

$$
T P u(x)=\sum_{\xi \in \mathbb{Z}^{n}} \int_{\mathbb{T}^{n}} \mathrm{e}^{\mathrm{i}(\phi(x, \xi)-z \cdot \xi)} c(x, z, \xi) u(z) \mathrm{d} z,
$$

where

$$
c(x, z, \xi)=\sum_{\eta \in \mathbb{Z}^{n}} \int_{\mathbb{T}^{n}} \mathrm{e}^{\mathrm{i}(y-z) \cdot(\eta-\xi)} a(x, y, \xi) p(y, \eta) \mathrm{d} y
$$

satisfies

$$
\left|\partial_{x}^{\alpha} \partial_{z}^{\beta} c(x, z, \xi)\right| \leq C_{\alpha \beta m t}\langle\xi\rangle^{m+\ell}
$$

for every $x, z \in \mathbb{T}^{n}, \xi \in \mathbb{Z}^{n}$ and $\alpha, \beta \in \mathbb{N}_{0}^{n}$. Moreover, we have an asymptotic expansion

$$
c(x, z, \xi) \sim \sum_{\alpha \geq 0} \frac{1}{\alpha !}\left(-D_{z}\right)^{(\alpha)}\left[a(x, z, \xi) \triangle_{\xi}^{\alpha} p(z, \xi)\right] .
$$

Furthermore, if $0 \leq \delta<\rho \leq 1, p \in S_{\rho, \delta}^{\ell}\left(\mathbb{T}^{n} \times \mathbb{Z}^{n}\right)$ and $a \in S_{\rho, \delta}^{m}\left(\mathbb{T}^{n} \times \mathbb{T}^{n} \times \mathbb{Z}^{n}\right)$, then $c \in S_{\rho, \delta}^{m+\ell}\left(\mathbb{T}^{n} \times \mathbb{T}^{n} \times \mathbb{Z}^{n}\right)$.

Remark 8.3. We note that if $T$ in (8.3) is a pseudo-differential operator with phase $\phi(x, \xi)=x \cdot \xi$ and amplitude $a(x, y, \xi)=a(x, \xi)$ independent of $y$, then the asymptotic expansion formula of two pseudo-differential operators $T \circ P$ becomes

$$
c(x, z, \xi) \sim \sum_{\alpha \geq 0} \frac{1}{\alpha !} a(x, \xi)\left(-D_{z}\right)^{(\alpha)} \triangle_{\xi}^{\alpha} p(z, \xi) .
$$

This is another representation for the composition compared to Theorem 4.3, but the amplitude of the composition here is a compound symbol.

Proof. Let us calculate the composition TP:

$$
\begin{aligned}
T P u(x) & =\sum_{\xi \in \mathbb{Z}^{n}} \int_{\mathbb{T}^{n}} \mathrm{e}^{\mathrm{i}(\phi(x, \xi)-y \cdot \xi)} a(x, y, \xi) P u(y) \mathrm{d} y \\
& =\sum_{\xi \in \mathbb{Z}^{n}} \int_{\mathbb{T}^{n}} \mathrm{e}^{\mathrm{i}(\phi(x, \xi)-y \cdot \xi)} a(x, y, \xi) \sum_{\eta \in \mathbb{Z}^{n}} \int_{\mathbb{T}^{n}} \mathrm{e}^{\mathrm{i}(y-z) \cdot \eta} p(y, \eta) u(z) \mathrm{d} z \mathrm{~d} y \\
& =\sum_{\xi \in \mathbb{Z}^{n}} \int_{\mathbb{T}^{n}} \mathrm{e}^{\mathrm{i}(\phi(x, \xi)-z \cdot \xi)} c(x, z, \xi) u(z) \mathrm{d} z,
\end{aligned}
$$

where

$$
c(x, z, \xi)=\sum_{\eta \in \mathbb{Z}^{n}} \int_{\mathbb{T}^{n}} \mathrm{e}^{\mathrm{i}(y-z) \cdot(\eta-\xi)} a(x, y, \xi) p(y, \eta) \mathrm{d} y .
$$

Denote $\theta:=\eta-\xi$, so that by the discrete Taylor formula as in Theorem 3.4, we formally get

$$
\begin{aligned}
c(x, z, \xi) & \sim \sum_{\theta \in \mathbb{Z}^{n}} \int_{\mathbb{T}^{n}} \mathrm{e}^{\mathrm{i}(y-z) \cdot \theta} a(x, y, \xi) \sum_{\alpha \geq 0} \frac{1}{\alpha !} \theta^{(\alpha)} \triangle_{\xi}^{\alpha} p(y, \xi) \mathrm{d} y \\
& =\sum_{\alpha \geq 0} \frac{1}{\alpha !} \sum_{\theta \in \mathbb{Z}^{n}} \int_{\mathbb{T}^{n}} \theta^{(\alpha)} \mathrm{e}^{\mathrm{i}(y-z) \cdot \theta} a(x, y, \xi) \triangle_{\xi}^{\alpha} p(y, \xi) \mathrm{d} y
\end{aligned}
$$




$$
=\left.\sum_{\alpha \geq 0} \frac{1}{\alpha !}\left(-D_{y}\right)^{(\alpha)}\left(a(x, y, \xi) \triangle_{\xi}^{\alpha} p(y, \xi)\right)\right|_{y=z} .
$$

Now we have to justify the asymptotic expansion. First we take a discrete Taylor expansion and using Theorem 3.4 we obtain

$$
p(y, \xi+\theta)=\sum_{|\omega|<M} \frac{1}{\omega !} \theta^{(\omega)} \triangle_{\xi}^{\omega} p(y, \xi)+r_{M}(y, \xi, \theta) .
$$

Let $Q(\theta)=\left\{\nu \in \mathbb{Z}^{n}:\left|\nu_{j}\right| \leq\left|\theta_{j}\right|\right.$ for all $\left.j=1, \ldots, n\right\}$ as in Theorem 3.4. Then

$$
\begin{aligned}
\left|\triangle_{\xi}^{\alpha} \partial_{y}^{\beta} r_{M}(y, \xi, \theta)\right| & \leq C\langle\theta\rangle^{M} \max _{\substack{|\omega|=M \\
\nu \in Q(\theta)}}\left|\triangle_{\xi}^{\alpha+\omega} \partial_{y}^{\beta} p(y, \xi+\nu)\right| \\
& \leq C\langle\theta\rangle^{M} \max _{\nu \in Q(\theta)}\langle\xi+\nu\rangle^{\ell-|\alpha|-M} \\
& \leq C\langle\theta\rangle^{M} \max _{\nu \in Q(\theta)}\langle\nu\rangle^{\ell \ell-|\alpha|-M \mid}\langle\xi\rangle^{\ell-|\alpha|-M} \\
& \leq C\langle\theta\rangle^{2 M+|\ell|+|\alpha|}\langle\xi\rangle^{\ell-|\alpha|-M} .
\end{aligned}
$$

The corresponding remainder term in the asymptotic expansion of $c(x, z, \xi)$ is

$$
\begin{aligned}
R_{M}(x, z, \xi) & =\sum_{\theta \in \mathbb{Z}^{n}} \int_{\mathbb{T}^{n}} \mathrm{e}^{\mathrm{i}(y-z) \cdot \theta} a(x, y, \xi) r_{M}(y, \xi, \theta) \mathrm{d} y \\
& =\sum_{\theta \in \mathbb{Z}^{n}} \int_{\mathbb{T}^{n}} \mathrm{e}^{\mathrm{i}(y-z) \cdot \theta}\langle\theta\rangle^{-2 N}\left(I-\mathscr{L}_{y}\right)^{N}\left[a(x, y, \xi) r_{M}(y, \xi, \theta)\right] \mathrm{d} y,
\end{aligned}
$$

where we integrated by parts exploiting that $\left(I-\mathscr{L}_{y}\right) \mathrm{e}^{\mathrm{i}(y-z) \cdot \theta}=\langle\theta\rangle^{2} \mathrm{e}^{\mathrm{i}(y-z) \cdot \theta}$, where $\mathscr{L}_{y}$ is the Laplacian with respect to $y$. Thus we get the estimate

$$
\left|\triangle_{\xi}^{\alpha} \partial_{x}^{\beta} \partial_{z}^{\gamma} R_{M}(x, z, \xi)\right| \leq C \sum_{\theta \in \mathbb{Z}^{n}}\langle\theta\rangle^{|\gamma|-2 N+2 M+|\ell|+|\alpha|}\langle\xi\rangle^{m+\ell-M}
$$

and we take $N \in \mathbb{N}$ so large that this sum over $\theta$ converges. Hence

$$
\left|\triangle_{\xi}^{\alpha} \partial_{x}^{\beta} \partial_{z}^{\gamma} R_{M}(x, z, \xi)\right| \leq C\langle\xi\rangle^{m+\ell-M} .
$$

This completes the proof of the first part of the theorem. Finally, we assume that $a \in S_{\rho, \delta}^{m}\left(\mathbb{T}^{n} \times \mathbb{T}^{n} \times \mathbb{Z}^{n}\right)$. Then also the terms in the asymptotic expansion and the remainder $R_{M}$ have corresponding decay properties in the $\xi$-differences, leading to the amplitude $c \in S_{\rho, \delta}^{m}\left(\mathbb{T}^{n} \times \mathbb{T}^{n} \times \mathbb{Z}^{n}\right)$. This completes the proof.

We now formulate the theorem about compositions of operators in the different order.

Theorem 8.4 (Composition $P(X, D) \circ T)$. Let $\phi: \mathbb{R}^{n} \times \mathbb{Z}^{n} \rightarrow \mathbb{R}$ be such that function $x \mapsto \mathrm{e}^{\mathrm{i} \phi(x, \xi)}$ is $2 \pi$-periodic for all $\xi \in \mathbb{Z}^{n}$. Let $T: C^{\infty}\left(\mathbb{T}^{n}\right) \rightarrow \mathcal{D}^{\prime}\left(\mathbb{T}^{n}\right)$ such that

$$
T u(x):=\sum_{\xi \in \mathbb{Z}^{n}} \int_{\mathbb{T}^{n}} \mathrm{e}^{\mathrm{i}(\phi(x, \xi)-y \cdot \xi)} a(x, y, \xi) u(y) \mathrm{d} y,
$$

where $a \in C^{\infty}\left(\mathbb{T}^{n} \times \mathbb{T}^{n} \times \mathbb{Z}^{n}\right)$ satisfies

$$
\left|\partial_{x}^{\alpha} \partial_{y}^{\beta} a(x, y, \xi)\right| \leq C_{\alpha \beta m}\langle\xi\rangle^{m}
$$


for every $x, y \in \mathbb{T}^{n}, \xi \in \mathbb{Z}^{n}$ and $\alpha, \beta \in \mathbb{N}_{0}^{n}$. Assume that for some $C>0$ we have

$$
C^{-1}\langle\xi\rangle \leq\left\langle\nabla_{x} \phi(x, \xi)\right\rangle \leq C\langle\xi\rangle
$$

for every $x \in \mathbb{T}^{n}, \xi \in \mathbb{Z}^{n}$, and that

$$
\left|\partial_{x}^{\alpha} \phi(x, \xi)\right| \leq C_{\alpha}\langle\xi\rangle, \quad\left|\partial_{x}^{\alpha} \triangle_{\xi}^{\beta} \phi(x, \xi)\right| \leq C_{\alpha \beta}
$$

for every $x \in \mathbb{T}^{n}, \xi \in \mathbb{Z}^{n}$ and $\alpha, \beta \in \mathbb{N}_{0}^{n}$ with $|\beta|=1$. Let $\widetilde{p} \in S^{\ell}\left(\mathbb{T}^{n} \times \mathbb{Z}^{n}\right)$ be a toroidal symbol and let $P=\widetilde{p}(X, D)$ be the corresponding pseudo-differential operator. Let $p(x, \xi)$ denote an extension of $\widetilde{p}(x, \xi)$ to a symbol in $S^{\ell}\left(\mathbb{T}^{n} \times \mathbb{R}^{n}\right)$ as given in Theorem 5.2. Then

$$
P T u(x)=\sum_{\xi \in \mathbb{Z}^{n}} \int_{\mathbb{R}^{n}} \mathrm{e}^{\mathrm{i}(\phi(x, \xi)-z \cdot \xi)} c(x, z, \xi) u(z) \mathrm{d} z,
$$

where

$$
\left|\partial_{x}^{\alpha} \partial_{z}^{\beta} c(x, z, \xi)\right| \leq C_{\alpha \beta}\langle\xi\rangle^{m+\ell}
$$

for every $x, z \in \mathbb{T}^{n}, \xi \in \mathbb{Z}^{n}$ and $\alpha, \beta \in \mathbb{N}_{0}^{n}$. Moreover,

$$
\left.\left.c(x, z, \xi) \sim \sum_{\alpha \geq 0} \frac{\mathrm{i}^{-|\alpha|}}{\alpha !} \partial_{\eta}^{\alpha} p(x, \eta)\right|_{\eta=\nabla_{x} \phi(x, \xi)} \partial_{y}^{\alpha}\left[\mathrm{e}^{\mathrm{i} \Psi(x, y, \xi)} a(y, z, \xi)\right]\right|_{y=x}
$$

where

$$
\Psi(x, y, \xi):=\phi(y, \xi)-\phi(x, \xi)+(x-y) \cdot \nabla_{x} \phi(x, \xi) .
$$

Let us make some remarks about quantities appearing in the asymptotic extension formula (8.6). It is geometrically reasonable to evaluate the symbol $\widetilde{p}(x, \xi)$ at the real flow generated by the phase function $\phi$ of the Fourier series operator $T$. This is the main complication compared with pseudo-differential operators for which we have Proposition 7.3. However, although a-priori symbol $\tilde{p}$ is defined only on $\mathbb{T}^{n} \times \mathbb{Z}^{n}$, we can still extend it to a symbol $p(x, \xi)$ on $\mathbb{T}^{n} \times \mathbb{R}^{n}$ by Theorem 5.2 , so that the restriction $\left.\partial_{\eta}^{\alpha} p(x, \eta)\right|_{\eta=\nabla_{x} \phi(x, \xi)}$ makes sense. We also note that function $\Psi(x, y, \xi)$ can not be in general considered as a function on $\mathbb{T}^{n} \times \mathbb{T}^{n} \times \mathbb{Z}^{n}$ because it may not be periodic in $x$ and $y$. However, we can still observe that the derivatives $\left.\partial_{y}^{\alpha}\left[\mathrm{e}^{\mathrm{i} \Psi(x, y, \xi)} a(y, z, \xi)\right]\right|_{y=x}$ are periodic in $x$ and $z$, so all terms in the right hand side of (8.6) are well-defined functions on $\mathbb{T}^{n} \times \mathbb{T}^{n} \times \mathbb{Z}^{n}$.

Finally, we note that if $\phi \in S_{\rho, \delta}^{1}\left(\mathbb{R}^{n} \times \mathbb{R}^{n}\right), \tilde{p} \in S_{\rho, \delta}^{\ell}\left(\mathbb{T}^{n} \times \mathbb{Z}^{n}\right), a \in S_{\rho, \delta}^{m}\left(\mathbb{T}^{n} \times \mathbb{T}^{n} \times \mathbb{Z}^{n}\right)$, and $0 \leq \delta<\rho \leq 1$, then we also have $c \in S_{\rho, \delta}^{m+\ell}\left(\mathbb{T}^{n} \times \mathbb{T}^{n} \times \mathbb{Z}^{n}\right)$.

Proof. To simplify the notation, let us drop writing tilde on $p$, and denote both symbols $\widetilde{p}$ and $p$ by the same letter $p$. There should be no confusion since they coincide on $\mathbb{T}^{n} \times \mathbb{Z}^{n}$. Let $P=p(X, D)$. We can write

$$
\begin{aligned}
P T u(x) & =\sum_{\eta \in \mathbb{Z}^{n}} \int_{\mathbb{T}^{n}} \mathrm{e}^{\mathrm{i}(x-y) \cdot \eta} p(x, \eta) T u(y) \mathrm{d} y \\
& =\sum_{\eta \in \mathbb{Z}^{n}} \int_{\mathbb{T}^{n}} \mathrm{e}^{\mathrm{i}(x-y) \cdot \eta} p(x, \eta) \sum_{\xi \in \mathbb{Z}^{n}} \int_{\mathbb{T}^{n}} \mathrm{e}^{\mathrm{i}(\phi(y, \xi)-z \cdot \xi)} a(y, z, \xi) u(z) \mathrm{d} z \mathrm{~d} y \\
& =\sum_{\xi \in \mathbb{Z}^{n}} \int_{\mathbb{T}^{n}} \mathrm{e}^{\mathrm{i}(\phi(x, \xi)-z \cdot \xi)} c(x, z, \xi) u(z) \mathrm{d} z,
\end{aligned}
$$


where

$$
c(x, z, \xi)=\sum_{\eta \in \mathbb{Z}^{n}} p(x, \eta) \int_{\mathbb{T}^{n}} \mathrm{e}^{\mathrm{i}(\phi(y, \xi)-\phi(x, \xi)+(x-y) \cdot \eta)} a(y, z, \xi) \mathrm{d} y .
$$

Let us fix some $x \in \mathbb{R}^{n}$, with corresponding equivalence class $[x] \in \mathbb{T}^{n}$ which we still denote by $x$. Let $V \subset \mathbb{R}^{n}$ be an open cube with side length equal to $2 \pi$ centred at $x$. Let $\chi=\chi(x, y) \in C^{\infty}\left(\mathbb{T}^{n} \times \mathbb{T}^{n}\right)$ be such that $0 \leq \chi \leq 1, \chi(x, y)=1$ for $|x-y|<\kappa$ for some sufficiently small $\kappa>0$, and such that $\operatorname{supp} \chi(x, \cdot) \cap \bar{V} \subset V$. The last condition means that $\left.\chi(x, \cdot)\right|_{V} \in C_{0}^{\infty}(V)$ is supported away from the boundaries of the cube $V$. Let

$$
\begin{aligned}
c(x, z, \xi) & =c^{I}(x, z, \xi)+c^{I I}(x, z, \xi), \text { where } \\
c^{I}(x, z, \xi) & =\sum_{\eta \in \mathbb{Z}^{n}} \int_{\mathbb{T}^{n}} \mathrm{e}^{\mathrm{i}(\phi(y, \xi)-\phi(x, \xi)+(x-y) \cdot \eta)}(1-\chi(x, y)) a(y, z, \xi) p(x, \eta) \mathrm{d} y, \\
c^{I I}(x, z, \xi) & =\sum_{\eta \in \mathbb{Z}^{n}} \int_{\mathbb{T}^{n}} \mathrm{e}^{\mathrm{i}(\phi(y, \xi)-\phi(x, \xi)+(x-y) \cdot \eta)} \chi(x, y) a(y, z, \xi) p(x, \eta) \mathrm{d} y .
\end{aligned}
$$

1. Estimate on the support of $1-\chi$. By making a decomposition into cones centred at $x$ viewed as a point in $\mathbb{R}^{n}$, it follows that we can assume without loss of generality that the support of $1-\chi$ is contained in a set where $C<\left|x_{j}-y_{j}\right|$, for some $1 \leq j \leq n$. In turn, because of the assumption on the support of $\left.\chi(x, \cdot)\right|_{V}$ it follows that $C<\left|x_{j}-y_{j}\right|<2 \pi-C$, for some $C>0$. Now we are going to apply the summation by parts formula (3.1) to estimate $c^{I}(x, z, \xi)$. First we notice that it follows that

$\triangle_{\eta_{j}} \mathrm{e}^{\mathrm{i}(x-y) \cdot \eta}=\mathrm{e}^{\mathrm{i}(x-y) \cdot\left(\eta+e_{j}\right)}-\mathrm{e}^{\mathrm{i}(x-y) \cdot \eta}=\mathrm{e}^{\mathrm{i}(x-y) \cdot \eta}\left(\mathrm{e}^{\mathrm{i}\left(x_{j}-y_{j}\right)}-1\right) \neq 0$ on $\operatorname{supp}(1-\chi)$.

Hence by the summation by parts formula (3.1) we get that

$$
\sum_{\eta \in \mathbb{Z}^{n}} \mathrm{e}^{\mathrm{i}(x-y) \cdot \eta} p(x, \eta)=\left(\mathrm{e}^{\mathrm{i}\left(x_{j}-y_{j}\right)}-1\right)^{-N_{1}} \sum_{\eta \in \mathbb{Z}^{n}} \mathrm{e}^{\mathrm{i}(x-y) \cdot \eta}{\overline{\triangle_{\eta_{j}}}}^{N_{1}} p(x, \eta)
$$

here sum on the right hand side converges absolutely for large enough $N_{1}$. On the other hand, we can integrate by parts with operator

$$
{ }^{t} L_{y}=\frac{1-\mathscr{L}_{y}}{\left\langle\nabla_{y} \phi(y, \xi)\right\rangle^{2}-\mathrm{i} \mathscr{L}_{y} \phi(y, \xi)},
$$

where $\mathscr{L}_{y}$ is the Laplace operator with respect to $y$, and for which we have $L_{y}^{N_{2}} \mathrm{e}^{\mathrm{i} \phi(y, \xi)}=$ $\mathrm{e}^{\mathrm{i} \phi(y, \xi)}$. Note that in view of our assumption (8.4) on $\phi$, we have

$$
\left|\left\langle\nabla_{y} \phi(y, \xi)\right\rangle^{2}-\mathrm{i} \mathscr{L}_{y} \phi(y, \xi)\right| \geq\left|\left\langle\nabla_{y} \phi(y, \xi)\right\rangle\right|^{2} \geq C_{1}\langle\xi\rangle^{2} .
$$

Therefore,

$$
\begin{aligned}
& c^{I}(x, z, \xi)=\sum_{\eta \in \mathbb{Z}^{n}} \int_{\mathbb{T}^{n}} \mathrm{e}^{\mathrm{i}(\phi(y, \xi)-\phi(x, \xi)+x \cdot \eta)} L_{y}^{N_{2}}\left\{\mathrm{e}^{-\mathrm{i} y \cdot \eta} \times\right. \\
&\left.\times\left(\mathrm{e}^{\mathrm{i}\left(x_{j}-y_{j}\right)}-1\right)^{-N_{1}} \bar{\triangle}_{\eta_{j}}{ }^{N_{1}} p(x, \eta)(1-\chi(x, y)) a(y, z, \xi)\right\} \mathrm{d} y .
\end{aligned}
$$


From the properties of amplitudes, we get

$$
\left|c^{I}(x, z, \xi)\right| \leq C \sum_{\eta \in \mathbb{Z}^{n}} \int_{V \cap\left\{2 \pi-c>\left|x_{j}-y_{j}\right|>c\right\}}\langle\xi\rangle^{m-2 N_{2}}\langle\eta\rangle^{2 N_{2}+\ell-N_{1}} \mathrm{~d} y \leq C\langle\xi\rangle^{-N}
$$

for all $N$, if we choose large enough $N_{2}$ and then large enough $N_{1}$. We can easily see that similar estimates work for the derivatives of $c^{I}$, completing the proof on the support of $1-\chi$.

2. Estimate on the support of $\chi$. Extending $\widetilde{p} \in S^{\ell}\left(\mathbb{T}^{n} \times \mathbb{Z}^{n}\right)$ to a symbol in $p \in S^{\ell}\left(\mathbb{T}^{n} \times \mathbb{R}^{n}\right)$ as given in Theorem 5.2 , we will make its usual Taylor expansion at $\eta=\nabla_{x} \phi(x, \xi)$, so that we have

$$
\begin{aligned}
p(x, \eta)= & \sum_{|\alpha|<M} \frac{\left(\eta-\nabla_{x} \phi(x, \xi)\right)^{\alpha}}{\alpha !} \partial_{\xi}^{\alpha} p\left(x, \nabla_{x} \phi(x, \xi)\right)+ \\
& +\sum_{|\alpha|=M} C_{\alpha}\left(\eta-\nabla_{x} \phi(x, \xi)\right)^{\alpha} r_{\alpha}\left(x, \xi, \eta-\nabla_{x} \phi(x, \xi)\right) \\
r_{\alpha}(x, \xi, \eta & \left.-\nabla_{x} \phi(x, \xi)\right)=\int_{0}^{1}(1-t)^{M_{1}} \partial_{\xi}^{\alpha} p\left(x, t \eta+(1-t) \nabla_{x} \phi(x, \xi)\right) \mathrm{d} t .
\end{aligned}
$$

Then

$$
c^{I I}(x, z, \xi)=\sum_{|\alpha|<M} \frac{1}{\alpha !} c_{\alpha}(x, z, \xi)+\sum_{|\alpha|=M} C_{\alpha} R_{\alpha}(x, z, \xi),
$$

where

$$
\begin{aligned}
& R_{\alpha}(x, z, \xi)= \sum_{\eta \in \mathbb{Z}^{n}} \int_{\mathbb{T}^{n}} \mathrm{e}^{\mathrm{i}(\phi(y, \xi)-\phi(x, \xi)+(x-y) \cdot \eta)}\left(\eta-\nabla_{x} \phi(x, \xi)\right)^{\alpha} \times \\
& \times \chi(x, y) r_{\alpha}\left(x, \xi, \eta-\nabla_{x} \phi(x, \xi)\right) a(y, z, \xi) \mathrm{d} y, \\
& c_{\alpha}(x, z, \xi)=\sum_{\eta \in \mathbb{Z}^{n}} \int_{\mathbb{T}^{n}} \mathrm{e}^{\mathrm{i}(\phi(y, \xi)-\phi(x, \xi)+(x-y) \cdot \eta)}\left(\eta-\nabla_{x} \phi(x, \xi)\right)^{\alpha} \times \\
& \times \chi(x, y) a(y, z, \xi) \partial_{\xi}^{\alpha} p\left(x, \nabla_{x} \phi(x, \xi)\right) \mathrm{d} y .
\end{aligned}
$$

Now using Corollary 6.6 we can calculate

$$
\begin{aligned}
c_{\alpha}(x, z, \xi)= & \left.\partial_{\xi}^{\alpha} p\left(x, \nabla_{x} \phi(x, \xi)\right)\left[D_{y}-\nabla_{x} \phi(x, \xi)\right]^{\alpha}\left\{\mathrm{e}^{\mathrm{i}(\phi(y, \xi)-\phi(x, \xi))} \chi(x, y) a(y, z, \xi)\right\}\right|_{y=x} \\
= & \partial_{\xi}^{\alpha} p\left(x, \nabla_{x} \phi(x, \xi)\right) \times \\
& \quad \times \int_{\mathbb{R}^{n}} \int_{V} \mathrm{e}^{\mathrm{i}(x-y) \cdot \eta}\left[\eta-\nabla_{x} \phi(x, \xi)\right]^{\alpha} \mathrm{e}^{\mathrm{i}(\phi(y, \xi)-\phi(x, \xi))} \chi(x, y) a(y, z, \xi) \mathrm{d} y \mathrm{~d} \eta \\
= & \left.\partial_{\xi}^{\alpha} p\left(x, \nabla_{x} \phi(x, \xi)\right) D_{y}^{\alpha}\left[\mathrm{e}^{\mathrm{i}\left(\phi(y, \xi)-\phi(x, \xi)+(x-y) \cdot \nabla_{x} \phi(x, \xi)\right.} \chi(x, y) a(y, z, \xi)\right]\right|_{y=x} .
\end{aligned}
$$

Since $\chi$ is identically equal to one for $y$ near $x$, we obtain the asymptotic formula (8.6), once the remainders $R_{\alpha}$ are analyzed.

3. Estimates for the remainder. Let us first write the remainder in the form

$$
\begin{array}{r}
R_{\alpha}(x, z, \xi)=\sum_{\eta \in \mathbb{Z}^{n}} \int_{\mathbb{T}^{n}} \mathrm{e}^{\mathrm{i}(x-y) \cdot \eta} r_{\alpha}\left(x, \xi, \eta-\nabla_{x} \phi(x, \xi)\right) \times \\
\times\left(\eta-\nabla_{x} \phi(x, \xi)\right)^{\alpha} \chi(x, y) g(y) \mathrm{d} y,
\end{array}
$$


with

$$
g(y)=\mathrm{e}^{\mathrm{i}(\phi(y, \xi)-\phi(x, \xi))} \chi(x, y) a(y, z, \xi),
$$

which is a $2 \pi$-periodic function of $y$. Now, we can use Corollary 6.6 to conclude that $R_{\alpha}(x, z, \xi)$ in (8.9) is equal to the periodization with respect to $x$ in the form $R_{\alpha}(x, z, \xi)=\mathcal{P}_{x} S_{\alpha}(x, z, \xi)$, where

$$
\begin{aligned}
S_{\alpha}(x, z, \xi)= & \left.r_{\alpha}\left(x, \xi, D_{y}-\nabla_{x} \phi(x, \xi)\right)\left(D_{y}-\nabla_{x} \phi(x, \xi)\right)^{\alpha} g(y)\right|_{y=x} \\
= & \int_{\mathbb{R}^{n}} \int_{V} \mathrm{e}^{\mathrm{i}(x-y) \cdot \eta} r_{\alpha}\left(x, \xi, \eta-\nabla_{x} \phi(x, \xi)\right) \times \\
& \times\left(\eta-\nabla_{x} \phi(x, \xi)\right)^{\alpha} \chi(x, y) g(y) \mathrm{d} y \mathrm{~d} \eta \\
= & \int_{\mathbb{R}^{n}} \int_{V} \mathrm{e}^{\mathrm{i}(x-y) \cdot \theta} \mathrm{e}^{\mathrm{i} \Psi(x, y, \xi)} \theta^{\alpha} \chi(x, y) a(y, z, \xi) r_{\alpha}(x, \xi, \theta) \mathrm{d} y \mathrm{~d} \theta,
\end{aligned}
$$

where we changed the variables by $\theta=\eta-\nabla_{x} \phi(x, \xi)$ and where

$$
\Psi(x, y, \xi):=\phi(y, \xi)-\phi(x, \xi)+(x-y) \cdot \nabla_{x} \phi(x, \xi)
$$

and

$$
r_{\alpha}(x, \xi, \theta)=\int_{0}^{1}(1-t)^{M_{1}} \partial_{\xi}^{\alpha} p\left(x, \nabla_{x} \phi(x, \xi)+t \theta\right) \mathrm{d} t .
$$

Since the periodization $\mathcal{P}_{x}$ does not change the orders in $z$ and $\xi$ it is enough to derive the required estimates for $S_{\alpha}(x, z, \xi)$.

Let $\rho \in C_{0}^{\infty}\left(\mathbb{R}^{n}\right)$ be such that $\rho(\theta)=1$ for $|\theta|<\epsilon / 2$, and $\rho(\theta)=0$ for $|\theta|>\epsilon$, for some small $\epsilon>0$ to be chosen later. We decompose

$$
\begin{aligned}
S_{\alpha}(x, z, \xi) & =S_{\alpha}^{I}(x, z, \xi)+S_{\alpha}^{I I}(x, z, \xi), \text { where } \\
S_{\alpha}^{I}(x, z, \xi)= & \int_{\mathbb{R}^{n}} \int_{v} \mathrm{e}^{\mathrm{i}(x-y) \cdot \theta} \rho\left(\frac{\theta}{\langle\xi\rangle}\right) \times \\
& \times r_{\alpha}(x, \xi, \theta) D_{y}^{\alpha}\left[\mathrm{e}^{\mathrm{i} \Psi(x, y, \xi)} \chi(x, y) a(y, z, \xi)\right] \mathrm{d} y \mathrm{~d} \theta \\
S_{\alpha}^{I I}(x, z, \xi)= & \int_{\mathbb{R}^{n}} \int_{V} \mathrm{e}^{\mathrm{i}(x-y) \cdot \theta}\left(1-\rho\left(\frac{\theta}{\langle\xi\rangle}\right)\right) \times \\
& \times r_{\alpha}(x, \xi, \theta) D_{y}^{\alpha}\left[\mathrm{e}^{\mathrm{i} \Psi(x, y, \xi)} \chi(x, y) a(y, z, \xi)\right] \mathrm{d} y \mathrm{~d} \theta .
\end{aligned}
$$

3.1. Estimate for $|\theta| \leq \epsilon\langle\xi\rangle$. For sufficiently small $\epsilon>0$, for any $0 \leq t \leq 1$, $\left\langle\nabla_{x} \phi(x, \xi)+t \theta\right\rangle$ and $\langle\xi\rangle$ are equivalent. Indeed, if we use the inequalities

$$
\langle z\rangle \leq 1+|z| \leq \sqrt{2}\langle z\rangle
$$

we get

$$
\begin{aligned}
\left\langle\nabla_{x} \phi(x, \xi)+t \theta\right\rangle & \leq\left(C_{2} \sqrt{2}+\epsilon\right)\langle\xi\rangle \\
\sqrt{2}\left\langle\nabla_{x} \phi(x, \xi)+t \theta\right\rangle & \geq 1+\left|\nabla_{x} \phi\right|-|\theta| \geq\left\langle\nabla_{x} \phi\right\rangle-|\theta| \geq\left(C_{1}-\epsilon\right)\langle\xi\rangle,
\end{aligned}
$$

so we will take $\epsilon<C_{1}$. This equivalence means that for $|\theta| \leq \epsilon\langle\xi\rangle$, function $r_{\alpha}(x, \xi, \theta)$ is dominated by $\langle\xi\rangle^{\ell-|\alpha|}$ since $p \in S^{\ell}\left(\mathbb{T}^{n} \times \mathbb{R}^{n}\right)$. We will need two auxiliary estimates. The first estimate 


$$
\begin{aligned}
\left|\partial_{\theta}^{\gamma}\left(\rho\left(\frac{\theta}{\langle\xi\rangle}\right) r_{\alpha}(x, \xi, \theta)\right)\right| & \leq C \sum_{\delta \leq \gamma}\left|\partial_{\theta}^{\delta} \rho\left(\frac{\theta}{\langle\xi\rangle}\right) \partial_{\theta}^{\gamma-\delta} r_{\alpha}(x, \xi, \theta)\right| \\
& \leq C \sum_{\delta \leq \gamma}\langle\xi\rangle^{-|\delta|}\langle\xi\rangle^{\ell-|\alpha|-|\gamma-\delta|} \\
& \leq C\langle\xi\rangle^{\ell-|\alpha|-|\gamma|}
\end{aligned}
$$

follows from the properties of $r_{\alpha}$. Before we state the second estimate, let us analyze the structure of $\partial_{y}^{\alpha} \mathrm{e}^{\mathrm{i} \Psi(x, y, \xi)}$. It has at most $|\alpha|$ powers of terms $\nabla_{y} \phi(y, \xi)-\nabla_{x} \phi(x, \xi)$, possibly also multiplied by at most $|\alpha|$ higher order derivatives $\partial_{y}^{\delta} \phi(y, \xi)$. The product of the terms of the form $\nabla_{y} \phi(y, \xi)-\nabla_{x} \phi(x, \xi)$ can be estimated by $C(|y-x|\langle\xi\rangle)^{|\alpha|}$. The terms containing no difference $\nabla_{y} \phi(y, \xi)-\nabla_{x} \phi(x, \xi)$ are the products of at most $|\alpha| / 2$ terms of the type $\partial_{y}^{\delta} \phi(y, \xi)$, and the product of all such terms can be estimated by $C\langle\xi\rangle^{|\alpha| / 2}$. Altogether, we obtain the estimate

$$
\left|\partial_{y}^{\alpha} \mathrm{e}^{\mathrm{i} \Psi(x, y, \xi)}\right| \leq C_{\alpha}(1+\langle\xi\rangle|y-x|)^{|\alpha|}\langle\xi\rangle^{|\alpha| / 2} .
$$

The second auxiliary estimate now is

$$
\left|D_{y}^{\alpha}\left[\mathrm{e}^{\mathrm{i} \Psi(x, y, \xi)} \chi(x, y) a(y, z, \xi)\right]\right| \leq C_{\alpha}(1+\langle\xi\rangle|y-x|)^{|\alpha|}\langle\xi\rangle^{\frac{|\alpha|}{2}+m} .
$$

Now we are ready to prove a necessary estimate for $S_{\alpha}^{I}(x, z, \xi)$. Let

$$
L_{\theta}=\frac{\left(1-\langle\xi\rangle^{2} \mathscr{L}_{\theta}\right)}{1+\langle\xi\rangle^{2}|x-y|^{2}}, \quad L_{\theta}^{N} \mathrm{e}^{\mathrm{i}(x-y) \cdot \theta}=\mathrm{e}^{\mathrm{i}(x-y) \cdot \theta},
$$

where $\mathscr{L}_{\theta}$ is the Laplace operator with respect to $\theta$. Integrations by parts with $L_{\theta}$ yield

$$
\begin{aligned}
S_{\alpha}^{I}(x, z, \xi)= & \int_{\mathbb{R}^{n}} \int_{V} \frac{\mathrm{e}^{\mathrm{i}(x-y) \cdot \theta}}{\left(1+\langle\xi\rangle^{2}|x-y|^{2}\right)^{N}}\left(1-\langle\xi\rangle^{2} \mathscr{L}_{\theta}\right)^{N} \\
& \left\{\rho\left(\frac{\theta}{\langle\xi\rangle}\right) r_{\alpha}(x, \xi, \theta) D_{y}^{\alpha}\left[\mathrm{e}^{\mathrm{i} \Psi(x, y, \xi)} \chi(x, y) a(y, z, \xi)\right]\right\} \mathrm{d} y \mathrm{~d} \theta \\
= & \int_{\mathbb{R}^{n}} \int_{V} \frac{\mathrm{e}^{\mathrm{i}(x-y) \cdot \theta}}{\left(1+\langle\xi\rangle^{2}|x-y|^{2}\right)^{N}} \sum_{|\gamma| \leq 2 N} C_{\gamma}\langle\xi\rangle^{|\gamma|} \\
& \left\{D_{y}^{\alpha}\left[\mathrm{e}^{\mathrm{i} \Psi(x, y, \xi)} \chi(x, y) a(y, z, \xi)\right] \partial_{\theta}^{\gamma}\left(\rho\left(\frac{\theta}{\langle\xi\rangle}\right) r_{\alpha}(x, \xi, \theta)\right)\right\} \mathrm{d} y \mathrm{~d} \theta .
\end{aligned}
$$

Using estimates (8.12), (8.13) and the fact that the measure of the support of function $\theta \mapsto \rho(\theta /\langle\xi\rangle)$ is estimated by $(\epsilon\langle\xi\rangle)^{n}$, we obtain the estimate

$$
\begin{aligned}
\left|S_{\alpha}^{I}(x, z, \xi)\right| & \leq C \sum_{|\gamma| \leq 2 N}\langle\xi\rangle^{n+|\gamma|+\frac{|\alpha|}{2}+m}\langle\xi\rangle^{\ell-|\alpha|-|\gamma|} \int_{V} \frac{(1+\langle\xi\rangle|y-x|)^{|\alpha|}}{\left(1+\langle\xi\rangle^{2}|x-y|^{2}\right)^{N}} \mathrm{~d} y \\
& \leq C\langle\xi\rangle^{\ell+m+n-\frac{|\alpha|}{2}}
\end{aligned}
$$

if we choose $N$ large enough, e.g. $N \geq M=|\alpha|$. 
Each derivative of $S_{\alpha}^{I}(x, z, \xi)$ with respect to $x$ or $\xi$ gives an extra power of $\theta$ under the integral. Integrating by parts, this amounts to taking more $y$-derivatives, giving a higher power of $\langle\xi\rangle$. However, this is not a problem if for the estimate for a given number of derivatives of the remainder $S_{\alpha}^{I}(x, z, \xi)$, we choose $M=|\alpha|$ sufficiently large.

3.2. Estimate for $|\theta|>\epsilon\langle\xi\rangle$. Let us define

$\omega(x, y, \xi, \theta)=(x-y) \cdot \theta+\Psi(x, y, \xi)=(x-y) \cdot\left(\nabla_{x} \phi(x, \xi)+\theta\right)+\phi(y, \xi)-\phi(x, \xi)$

From (8.4) and (8.5) we have

$$
\begin{aligned}
& \left|\nabla_{y} \omega\right|=\left|-\theta+\nabla_{y} \phi-\nabla_{x} \phi\right| \leq 2 C_{2}(|\theta|+\langle\xi\rangle) \\
& \left|\nabla_{y} \omega\right| \geq|\theta|-\left|\nabla_{y} \phi-\nabla_{x} \phi\right| \geq \frac{1}{2}|\theta|+\left(\frac{\epsilon}{2}-C_{0}|x-y|\right)\langle\xi\rangle \geq C(|\theta|+\langle\xi\rangle),
\end{aligned}
$$

if we choose $\kappa<\frac{\epsilon}{2 C_{0}}$, since $|x-y|<\kappa$ in the support of $\chi$ in $V$ (recall that we were free to choose $\kappa>0$ ). Let us denote

$$
\sigma_{\gamma_{1}}(x, y, \xi):=\mathrm{e}^{-\mathrm{i} \Psi(x, y, \xi)} D_{y}^{\gamma_{1}} \mathrm{e}^{\mathrm{i} \Psi(x, y, \xi)}
$$

For any $\nu$ we have an estimate

$$
\left|\partial_{y}^{\nu} \sigma_{\gamma_{1}}(x, y, \xi)\right| \leq C\langle\xi\rangle^{\left|\gamma_{1}\right|}
$$

because of our assumption (8.5) that $\left|\partial_{y}^{\nu} \phi(y, \xi)\right| \leq C_{\nu}\langle\xi\rangle$. For $M=|\alpha|>\ell$ we also observe that

$$
\left|r_{\alpha}(x, \xi, \theta)\right| \leq C_{\alpha},\left|\partial_{y}^{\nu} a(y, z, \xi)\right| \leq C_{\beta}\langle\xi\rangle^{m}
$$

Let us take ${ }^{t} L_{y}=\mathrm{i}\left|\nabla_{y} \omega\right|^{-2} \sum_{j=1}^{n}\left(\partial_{y_{j}} \omega\right) \partial_{y_{j}}$. It can be shown by induction that operator $L_{y}^{N}$ has the form

$$
L_{y}^{N}=\frac{1}{\left|\nabla_{y} \omega\right|^{4 N}} \sum_{|\nu| \leq N} P_{\nu, N} \partial_{y}^{\nu}, \quad P_{\nu, N}=\sum_{|\mu|=2 N} c_{\nu \mu \delta_{j}}\left(\nabla_{y} \omega\right)^{\mu} \partial_{y}^{\delta_{1}} \omega \cdots \partial_{y}^{\delta_{N}} \omega
$$

where $|\mu|=2 N,\left|\delta_{j}\right| \geq 1, \sum_{j=1}^{N}\left|\delta_{j}\right|+|\nu|=2 N$. It follows from (8.5) and (8.14) that $\left|P_{\nu, N}\right| \leq C(|\theta|+\langle\xi\rangle)^{3 N}$, since for all $\delta_{j}$ we have $\left|\partial_{y}^{\delta_{j}} \omega\right| \leq C(|\theta|+\langle\xi\rangle)$. By the Leibnitz 
formula we have

$$
\begin{aligned}
S_{\alpha}^{I I}(x, z, \xi)= \\
=\int_{\mathbb{R}^{n}} \int_{V} \mathrm{e}^{\mathrm{i}(x-y) \cdot \theta}\left(1-\rho\left(\frac{\theta}{\langle\xi\rangle}\right)\right) r_{\alpha}(x, \xi, \theta) \times \\
\quad \times D_{y}^{\alpha}\left[\mathrm{e}^{\mathrm{i} \Psi(x, y, \xi)} \chi(x, y) a(y, z, \xi)\right] \mathrm{d} y \mathrm{~d} \theta \\
=\int_{\mathbb{R}^{n}} \int_{V} \mathrm{e}^{\mathrm{i} \omega(x, y, \xi, \theta)}\left(1-\rho\left(\frac{\theta}{\langle\xi\rangle}\right)\right) r_{\alpha}(x, \xi, \theta) \times \\
\quad \times \sum_{\gamma_{1}+\gamma_{2}+\gamma_{3}=\alpha} \sigma_{\gamma_{1}}(x, y, \xi) D_{y}^{\gamma_{2}} \chi(x, y) D_{y}^{\gamma_{3}} a(y, z, \xi) \mathrm{d} y \mathrm{~d} \theta \\
=\int_{\mathbb{R}^{n}} \int_{V} \mathrm{e}^{\mathrm{i} \omega(x, y, \xi, \theta)}\left|\nabla_{y} \omega\right|^{-4 N} \sum_{|\nu| \leq N} P_{\nu, N}(x, y, \xi, \theta)\left(1-\rho\left(\frac{\theta}{\langle\xi\rangle}\right)\right) \times \\
\quad \times r_{\alpha}(x, \xi, \theta) \sum_{\gamma_{1}+\gamma_{2}+\gamma_{3}=\alpha} \partial_{y}^{\nu}\left(\sigma_{\gamma_{1}}(x, y, \xi) D_{y}^{\gamma_{2}} \chi(x, y) D_{y}^{\gamma_{3}} a(y, z, \xi)\right) \mathrm{d} y \mathrm{~d} \theta .
\end{aligned}
$$

It follows now from (8.15) and (8.16) that

$$
\begin{aligned}
\left|S_{\alpha}^{I I}(x, z, \xi)\right| & \leq C \int_{|\theta|>\epsilon \in \xi\rangle / 2}(|\theta|+\langle\xi\rangle)^{-N}\langle\xi\rangle^{|\alpha|}\langle\xi\rangle^{m} \mathrm{~d} \theta \\
& \leq C\langle\xi\rangle^{m+|\alpha|+n-N},
\end{aligned}
$$

which yields the desired estimate if we take large enough $N$. For the derivatives of $S_{\alpha}^{I I}(x, z, \xi)$, similar to Part 3.1 for $S_{\alpha}^{I}$, we can get extra powers of $\theta$, which can be taken care of by choosing large $N$. The proof of Theorem 8.4 is now complete.

Remark 8.5. We could also use the following asymptotic expansion for $c$ based on the discrete Taylor expansion from Theorem 3.4:

$$
\begin{aligned}
c(x, z, \xi) & \sim \sum_{\theta \in \mathbb{Z}^{n}} \sum_{\alpha \geq 0} \frac{1}{\alpha !} \theta^{(\alpha)}\left[\triangle_{\omega}^{\alpha} p(x, \omega)\right]_{\omega=\nabla_{x} \phi(x, \xi)} \int_{\mathbb{T}^{n}} \mathrm{e}^{\mathrm{i}(\Psi(x, y, \xi)+(x-y) \cdot \theta)} a(y, z, \xi) \mathrm{d} y \\
& =\sum_{\alpha \geq 0} \frac{1}{\alpha !}\left[\triangle_{\omega}^{\alpha} p(x, \omega)\right]_{\omega=\nabla_{x} \phi(x, \xi)} \sum_{\theta \in \mathbb{Z}^{n}} \int_{\mathbb{T}^{n}} \theta^{(\alpha)} \mathrm{e}^{\mathrm{i}(x-y) \cdot \theta} \mathrm{e}^{\mathrm{i} \Psi(x, y, \xi)} a(y, z, \xi) \mathrm{d} y \\
& =\sum_{\alpha \geq 0} \frac{1}{\alpha !}\left[\triangle_{\omega}^{\alpha} p(x, \omega)\right]_{\omega=\nabla_{x} \phi(x, \xi)} D_{y}^{(\alpha)}\left[\mathrm{e}^{\mathrm{i} \Psi(x, y, \xi)} a(y, z, \xi)\right]_{y=x} .
\end{aligned}
$$

\section{CONDitions For $L^{2}$-BOUndedness}

In this section we will discuss what conditions on the toroidal symbol a guarantee the $L^{2}$-boundedness of the corresponding pseudo-differential operator $a(X, D)$ : $C^{\infty}\left(\mathbb{T}^{n}\right) \rightarrow \mathcal{D}^{\prime}\left(\mathbb{T}^{n}\right)$. In Theorem 9.2 we will also prove a result on the $L^{2}$-boundedness of Fourier series operators, and in Remark 9.4 we will give its consequence for the boundedness in Sobolev spaces.

Theorem 9.1. Let $a: \mathbb{T}^{n} \times \mathbb{Z}^{n} \rightarrow \mathbb{C}$ be such that

$$
\left|\partial_{x}^{\beta} a(x, \xi)\right| \leq C \quad \text { for all }(x, \xi) \in \mathbb{T}^{n} \times \mathbb{Z}^{n},
$$

and all $|\beta| \leq n+1$. Then operator $a(X, D)$ extends to a bounded operator on $L^{2}\left(\mathbb{T}^{n}\right)$. 
We note that compared with several well-known theorems on the $L^{2}$-boundedness of pseudo-differential operators (see e.g. Calderon and Vaillancourt [8], Coifman and Meyer [9], Cordes [10]), Theorem 9.1 does not require any regularity with respect to the $\xi$-variable. In fact, the boundedness of all partial differences of all orders $\geq 1$ with respect to $\xi$ follows automatically from (9.1).

Proof. We can write

$$
\begin{aligned}
a(X, D) f(x) & =\sum_{\xi \in \mathbb{Z}^{n}} a(x, \xi)\left(\mathscr{F}_{\mathbb{T}^{n}} f\right)(\xi) \mathrm{e}^{\mathrm{i} x \cdot \xi} \\
& =\sum_{\xi, \eta \in \mathbb{Z}^{n}}\left(\mathscr{F}_{\mathbb{T}^{n}} a\right)(\eta, \xi)\left(\mathscr{F}_{\mathbb{T}^{n}} f\right)(\xi) \mathrm{e}^{\mathrm{i} x \cdot(\xi+\eta)} \\
& =\sum_{\omega \in \mathbb{Z}^{n}} \mathrm{e}^{\mathrm{i} x \cdot \omega} \sum_{\xi \in \mathbb{Z}^{n}}\left(\mathscr{F}_{\mathbb{T}^{n}} a\right)(\omega-\xi, \xi)\left(\mathscr{F}_{\mathbb{T}^{n}} f\right)(\xi),
\end{aligned}
$$

where $\left(\mathscr{F}_{\mathbb{T}^{n}} a\right)(\eta, \xi)$ is the $\eta^{\text {th }}$ Fourier coefficient of $a(\cdot, \xi)$. Here $\left|\left(\mathscr{F}_{\mathbb{T}^{n}} a\right)(\eta, \xi)\right| \leq$ $C\langle\eta\rangle^{-n-1}$, so that

$$
\begin{aligned}
\|a(X, D) f\|_{L^{2}\left(\mathbb{T}^{n}\right)}^{2} & =\int_{\mathbb{T}^{n}}|a(X, D) f(x)|^{2} \mathrm{~d} x \\
& =\sum_{\omega \in \mathbb{Z}^{n}}\left|\mathscr{F}_{\mathbb{T}^{n}}(a(X, D) f)(\omega)\right|^{2} \\
& =\sum_{\omega \in \mathbb{Z}^{n}}\left|\sum_{\xi \in \mathbb{Z}^{n}}\left(\mathscr{F}_{\mathbb{T}^{n}} a\right)(\omega-\xi, \xi)\left(\mathscr{F}_{\mathbb{T}^{n}} f\right)(\xi)\right|^{2} \\
\leq & \left(\sup _{\omega \in \mathbb{Z}^{n}} \sum_{\xi \in \mathbb{Z}^{n}}\left|\left(\mathscr{F}_{\mathbb{T}^{n}} a\right)(\omega-\xi, \xi)\right|\right) \times \\
& \times\left(\sup _{\xi \in \mathbb{Z}^{n}} \sum_{\omega \in \mathbb{Z}^{n}}\left|\left(\mathscr{F}_{\mathbb{T}^{n}} a\right)(\omega-\xi, \xi)\right|\right) \sum_{\xi \in \mathbb{Z}^{n}}\left|\left(\mathscr{F}_{\mathbb{T}^{n}} f\right)(\xi)\right|^{2} \\
\leq & C\|f\|_{L^{2}\left(\mathbb{T}^{n}\right)}^{2}
\end{aligned}
$$

by the discrete version of Young's inequality.

As an extension of this result to the setting of Fourier series operators, we have the following $L^{2}$-boundedness property.

Theorem 9.2. Let $T: C^{\infty}\left(\mathbb{T}^{n}\right) \rightarrow \mathcal{D}^{\prime}\left(\mathbb{T}^{n}\right)$ be defined by

$$
T u(x)=\sum_{k \in \mathbb{Z}^{n}} \mathrm{e}^{\mathrm{i} \phi(x, k)} a(x, k)\left(\mathscr{F}_{\mathbb{T}^{n}} u\right)(k),
$$

where $\phi: \mathbb{R}^{n} \times \mathbb{Z}^{n} \rightarrow \mathbb{R}$ and $a: \mathbb{T}^{n} \times \mathbb{Z}^{n} \rightarrow \mathbb{C}$. Assume that function $x \mapsto \mathrm{e}^{\mathrm{i} \phi(x, \xi)}$ is $2 \pi$-periodic for every $\xi \in \mathbb{Z}^{n}$, and that for all $|\alpha| \leq 2 n+1$ and $|\beta|=1$ we have

$$
\left|\partial_{x}^{\alpha} a(x, k)\right| \leq C \text { and }\left|\partial_{x}^{\alpha} \triangle_{k}^{\beta} \phi(x, k)\right| \leq C \quad \text { for all }(x, k) \in \mathbb{T}^{n} \times \mathbb{Z}^{n} .
$$

Assume also that

$$
\left|\nabla_{x} \phi(x, k)-\nabla_{x} \phi(x, l)\right| \geq C|k-l| \text { for all } x \in \mathbb{T}^{n}, k, l \in \mathbb{Z}^{n} .
$$


Then $T$ extends to a bounded operator on $L^{2}\left(\mathbb{T}^{n}\right)$.

Note that condition (9.3) is a discrete version of the usual local graph condition for Fourier integral operators, necessary for the local $L^{2}$-boundedness. We also note that conditions on the boundedness of higher order differences of phase and amplitude would follow automatically from condition (9.2). Therefore, this theorem relaxes assumptions on the behaviour with respect to the dual variable, compared, for example, with the corresponding result by Ruzhansky and Sugimoto [20].

Proof. Since for $u: \mathbb{T}^{n} \rightarrow \mathbb{C}$ we have $\|u\|_{L^{2}\left(\mathbb{T}^{n}\right)}=\left\|\mathscr{F}_{\mathbb{T}^{n}} u\right\|_{\ell^{2}\left(\mathbb{Z}^{n}\right)}$, it is enough to prove that operator

$$
S w(x)=\sum_{k \in \mathbb{Z}^{n}} \mathrm{e}^{\mathrm{i} \phi(x, k)} a(x, k) w(k)
$$

is bounded from $\ell^{2}\left(\mathbb{Z}^{n}\right)$ to $L^{2}\left(\mathbb{T}^{n}\right)$. Let us define $S_{l} w(x)=\mathrm{e}^{\mathrm{i} \phi(x, l)} a(x, l) w(l)$, so that $S=\sum_{l \in \mathbb{Z}^{n}} S_{l}$. From the identity

$$
\left(w, S^{*} v\right)_{\ell^{2}\left(\mathbb{Z}^{n}\right)}=(S w, v)_{L^{2}\left(\mathbb{T}^{n}\right)}=\int_{\mathbb{T}^{n}} \sum_{k \in \mathbb{Z}^{n}} \mathrm{e}^{\mathrm{i} \phi(x, k)} a(x, k) w(k) \overline{v(x)} \mathrm{d} x
$$

we find that the adjoint $S^{*}$ to $S$ is given by

$$
\left(S^{*} v\right)(k)=\int_{\mathbb{T}^{n}} \mathrm{e}^{-\mathrm{i} \phi(x, k)} \overline{a(x, k)} v(x) \mathrm{d} x
$$

and so we also have

$$
\left(S_{l}^{*} v\right)(m)=\delta_{l m} \int_{\mathbb{T}^{n}} \mathrm{e}^{-\mathrm{i} \phi(x, m)} \overline{a(x, m)} v(x) \mathrm{d} x=\delta_{l m}\left(S^{*} v\right)(l) .
$$

It follows that

$$
\begin{aligned}
S_{k} S_{l}^{*} v(x) & =\mathrm{e}^{\mathrm{i} \phi(x, k)} a(x, k)\left(S_{l}^{*} v\right)(k) \\
& =\delta_{l k} \int_{\mathbb{T}^{n}} \mathrm{e}^{\mathrm{i} \phi(x, k)} a(x, k) \mathrm{e}^{-\mathrm{i} \phi(y, k)} \overline{a(y, k)} v(y) \mathrm{d} y \\
& =\int_{\mathbb{T}^{n}} K_{k l}(x, y) v(y) \mathrm{d} y
\end{aligned}
$$

where $K_{k l}(x, y)=\delta_{k l} \mathrm{e}^{\mathrm{i}[\phi(x, k)-\phi(y, k)]} a(x, k) \overline{a(y, k)}$. From (9.2) and compactness of the torus it follows that the kernel $K_{k l}$ is bounded and that $\left\|S_{k} S_{l}^{*} v\right\|_{L^{2}\left(\mathbb{T}^{n}\right)} \leq C \delta_{k l}\|v\|_{L^{2}\left(\mathbb{T}^{n}\right)}$. In particular, we can trivially conclude that for any $N \geq 0$ we have the estimate

$$
\left\|S_{k} S_{l}^{*}\right\|_{L^{2}\left(\mathbb{T}^{n}\right) \rightarrow L^{2}\left(\mathbb{T}^{n}\right)} \leq \frac{C_{N}}{1+|k-l|^{N}}
$$

On the other hand, we have

$$
\begin{aligned}
\left(S_{l}^{*} S_{k} w\right)(m) & =\delta_{l m} \int_{\mathbb{T}^{n}} \mathrm{e}^{-\mathrm{i} \phi(x, l)} \overline{a(x, l)}\left(S_{k} w\right)(x) \mathrm{d} x \\
& =\delta_{l m} \int_{\mathbb{T}^{n}} \mathrm{e}^{\mathrm{i}[\phi(x, k)-\phi(x, l)]} a(x, k) \overline{a(x, l)} w(k) \mathrm{d} x \\
& =\sum_{\mu \in \mathbb{Z}^{n}} \overline{K_{l k}}(m, \mu) w(\mu),
\end{aligned}
$$


where $\widetilde{K_{l k}}(m, \mu)=\delta_{l m} \delta_{k \mu} \int_{\mathbb{T}^{n}} \mathrm{e}^{\mathrm{i}[\phi(x, k)-\phi(x, l)]} a(x, k) \overline{a(x, l)} \mathrm{d} x$. Now, if $k \neq l$, integrating by parts $(2 n+1)$-times with operator $\frac{1}{\mathrm{i}} \frac{\nabla_{x} \phi(x, k)-\nabla_{x} \phi(x, l)}{\left|\nabla_{x} \phi(x, k)-\nabla_{x} \phi(x, l)\right|^{2}} \cdot \nabla_{x}$ and using the periodicity of $a$ and $\nabla_{x} \phi$ (so there are no boundary terms), we get the estimate

$$
\left|\widetilde{K_{l k}}(m, \mu)\right| \leq \frac{C \delta_{l m} \delta_{k \mu}}{1+|k-l|^{2 n+1}}
$$

where we also used that by the discrete Taylor theorem 3.4 the second condition in (9.2) implies that

$$
\left|\nabla_{x} \phi(x, k)-\nabla_{x} \phi(x, l)\right| \leq C|k-l| \text { for all } x \in \mathbb{T}^{n}, k, l \in \mathbb{Z}^{n} .
$$

Estimate (9.5) implies

$$
\sup _{m} \sum_{\mu}\left|\widetilde{K_{l k}}(m, \mu)\right|=\left|\widetilde{K_{l k}}(l, k)\right| \leq \frac{C}{1+|k-l|^{2 n+1}},
$$

and similarly for $\sup _{\mu} \sum_{m}$, so that we have

$$
\left\|S_{l}^{*} S_{k}\right\|_{\ell^{2}\left(\mathbb{Z}^{n}\right) \rightarrow \ell^{2}\left(\mathbb{Z}^{n}\right)} \leq \frac{C}{1+|k-l|^{2 n+1}} .
$$

These estimates for norms $\left\|S_{k} S_{l}^{*}\right\|_{L^{2}\left(\mathbb{T}^{n}\right) \rightarrow L^{2}\left(\mathbb{T}^{n}\right)}$ and $\left\|S_{l}^{*} S_{k}\right\|_{\ell^{2}\left(\mathbb{Z}^{n}\right) \rightarrow \ell^{2}\left(\mathbb{Z}^{n}\right)}$ in (9.4) and (9.6), respectively, imply the theorem by a modification of Cotlar's lemma given in Proposition 9.3, which we use with $\mathcal{H}=\ell^{2}\left(\mathbb{Z}^{n}\right)$ and $\mathcal{G}=L^{2}\left(\mathbb{T}^{n}\right)$.

The following statement is a modification of the well-known Cotlar's lemma taking into account the fact the operators in our application act on functions on different Hilbert spaces. The proof follows [28, p. 280] but there is a difference in how we estimate operator norms because we cannot immediately replace operator $S$ by $S^{*} S$ in the estimates since they act on functions on different spaces. We omit the proof.

Proposition 9.3 (Variant of Cotlar's lemma). Let $\mathcal{H}, \mathcal{G}$ be Hilbert spaces. Assume that a family of bounded linear operators $\left\{S_{j}: \mathcal{H} \rightarrow \mathcal{G}\right\}_{j \in \mathbb{Z}^{r}}$ and positive constants $\{\gamma(j)\}_{j \in \mathbb{Z}^{r}}$ satisfy

$$
\left\|S_{l}^{*} S_{k}\right\|_{\mathcal{H} \rightarrow \mathcal{H}} \leq[\gamma(l-k)]^{2}, \quad\left\|S_{l} S_{k}^{*}\right\|_{\mathcal{G} \rightarrow \mathcal{G}} \leq[\gamma(l-k)]^{2},
$$

and $A=\sum_{j \in \mathbb{Z}^{r}} \gamma(j)<\infty$. Then the operator $S=\sum_{j \in \mathbb{Z}^{r}} S_{j}$ satisfies $\|S\|_{\mathcal{H} \rightarrow \mathcal{G}} \leq A$.

Remark 9.4 (Sobolev spaces). By using pseudo-differential operator $\mathscr{L}_{s}(D)$ with toroidal symbol $\left(1+|\xi|^{2}\right)^{s / 2} \in S_{1,0}^{s}\left(\mathbb{T}^{n} \times \mathbb{Z}^{n}\right), s \in \mathbb{R}$, we can define Sobolev spaces $H^{s}\left(\mathbb{T}^{n}\right)$ on the torus as spaces of all $f \in \mathcal{D}^{\prime}\left(\mathbb{T}^{n}\right)$ such that $\mathscr{L}_{s}(D) f \in L^{2}\left(\mathbb{T}^{n}\right)$. Then using Theorems 8.2, 8.4, and 9.2, we obtain the result on the boundedness of Fourier series operators on Sobolev spaces. Namely, let $T$ be a Fourier series operator as in Theorem 8.4. Then $T$ is bounded on $H^{s}\left(\mathbb{T}^{n}\right)$ for all $s \in \mathbb{R}$.

\section{Applications to hyperbolic equations}

In this section we will briefly discuss how the analysis can be applied to construct global parametrices for the hyperbolic equations on the torus and how to embed certain problems in $\mathbb{R}^{n}$ into the torus. As already mentioned in the introduction, the finite propagation speed of singularities in hyperbolic equations allows one to cut-off the equation and the Cauchy data for large $x$ for the local analysis of singularities of 
solutions for bounded times. Then the problem can be embedded into $\mathbb{T}^{n}$ in order to apply the periodic analysis developed in this paper. One of the advantages of this procedure is that since phases and amplitudes now are only evaluated at $\xi \in \mathbb{Z}^{n}$ one can apply this also for problems with low regularity in $\xi$, in particular to problems for weakly hyperbolic equations or systems with variable multiplicities. For example, if the principal part has constant coefficients then the loss of regularity occurs only in $\xi$ so techniques developed in this paper can be applied.

Let $a(X, D)$ be a pseudo-differential operator with symbol $a=a(x, \xi) \in S^{m}\left(\mathbb{R}^{n} \times\right.$ $\mathbb{R}^{n}$ ) (with some properties to be specified). There is no difference in the subsequent argument if $a=a(t, x, \xi)$ also depends on $t$. For a function $u=u(t, x)$ of $t \in \mathbb{R}$ and $x \in \mathbb{R}^{n}$ we write

$$
\begin{aligned}
a(X, D) u(t, x) & =\int_{\mathbb{R}^{n}} a(x, \xi)\left(\mathscr{F}_{\mathbb{R}^{n}} u\right)(t, \xi) \mathrm{e}^{\mathrm{i} x \cdot \xi} \mathrm{d} \xi \\
& =\int_{\mathbb{R}^{n}} \int_{\mathbb{R}^{n}} \mathrm{e}^{\mathrm{i}(x-y) \cdot \xi} a(x, \xi) u(t, y) \mathrm{d} y \mathrm{~d} \xi .
\end{aligned}
$$

Let $u(t, \cdot) \in L^{1}\left(\mathbb{R}^{n}\right)\left(0<t<t_{0}\right)$ be a solution to the hyperbolic problem

$$
\left\{\begin{array}{l}
\mathrm{i} \frac{\partial}{\partial t} u(t, x)=a(X, D) u(t, x), \\
u(0, x)=f(x),
\end{array}\right.
$$

where $f \in L^{1}\left(\mathbb{R}^{n}\right)$ is compactly supported.

Assume now that $a(X, D)=a_{1}(X, D)+a_{0}(X, D)$ where $a_{1}(x, \xi)$ is $2 \pi$-periodic and $a_{0}(x, \xi)$ is compactly supported in $x$ (assume even that $\left.\operatorname{supp} a_{0}(\cdot, \xi) \subset[-\pi, \pi]^{n}\right)$. A simple example is a constant coefficient symbol $a_{1}(x, \xi)=a_{1}(\xi)$. Let us also assume that supp $f \subset[-\pi, \pi]^{n}$.

We will now describe a way to periodize problem (10.1). According to Proposition 6.7, we can replace (10.1) by

$$
\left\{\begin{array}{l}
\mathrm{i} \frac{\partial}{\partial t} u(t, x)=\left(a_{1}(x, D)+\left(\mathcal{P} a_{0}\right)(X, D)\right) u(t, x)+R u(t, x), \\
u(x, 0)=f(x),
\end{array}\right.
$$

where the symbol $a_{1}+\mathcal{P} a_{0}$ is periodic and $R$ is a smoothing operator. To study singularities of (10.1), it is sufficient to analyze the Cauchy problem

$$
\left\{\begin{array}{l}
\mathrm{i} \frac{\partial}{\partial t} v(t, x)=\left(a_{1}(x, D)+\left(\mathcal{P} a_{0}\right)(X, D)\right) v(t, x), \\
v(x, 0)=f(x)
\end{array}\right.
$$

since by Duhamel's formula we have $\mathrm{WF}(u-v)=\emptyset$. This problem can be transferred to the torus. Let $w(t, x)=\mathcal{P} v(\cdot, t)(x)$. By Theorem 6.4 it solves the Cauchy problem

$$
\left\{\begin{array}{l}
\mathrm{i} \frac{\partial}{\partial t} w(t, x)=\left(\widetilde{a_{1}}(x, D)+\widetilde{\mathcal{P} a_{0}}(X, D)\right) w(t, x), \\
w(x, 0)=\mathcal{P} f(x)
\end{array}\right.
$$

Now, if $a \in S^{1}$ is of the first order, the calculus constructed in previous sections yields the solution in the form

$$
w(t, x) \equiv T_{t} f(x)=\sum_{k \in \mathbb{Z}^{n}} \mathrm{e}^{\mathrm{i} \phi(t, x, k)} b(t, x, k) \mathscr{F}_{\mathbb{T}^{n}}(\mathcal{P} f)(k),
$$


where $\phi(t, x, \xi)$ and $b(t, x, \xi)$ satisfy discrete analogues of the eikonal and transport equations. Here we note that $\mathscr{F}_{\mathbb{T}^{n}}(\mathcal{P} f)(k)=\left(\mathscr{F}_{\mathbb{R}^{n}} f\right)(k)$. We also note that in principal the phase $\phi(t, x, k)$ is defined for discrete values of $k \in \mathbb{Z}^{n}$, so there is no issue of regularity, making this representation potentially applicable to low regularity problems and weakly hyperbolic equations.

Let us give a short example. If the symbol $a_{1}(x, \xi)=a_{1}(\xi)$ has constant coefficients and belongs to $S^{1}$, and $a_{0}$ belongs to $S^{0}$, we can find that the phase is given by $\phi(t, x, k)=x \cdot k+t a_{1}(k)$. In particular, $\nabla_{x} \phi(x, k)=k$. Applying $a(X, D)$ to $w(t, x)=$ $T_{t} f(x)$ and using the composition formula from Theorem 8.4 we obtain

$$
a(X, D) T_{t} f(x)=\sum_{k \in \mathbb{Z}^{n}} \int_{\mathbb{R}^{n}} \mathrm{e}^{\mathrm{i}\left((x-z) \cdot k+t a_{1}(k)\right)} c(t, x, k) f(z) \mathrm{d} z,
$$

where

$$
\left.c(t, x, k) \sim \sum_{\alpha \geq 0} \frac{\mathrm{i}^{-|\alpha|}}{\alpha !} \partial_{\xi}^{\alpha} a(x, \xi)\right|_{\xi=k} \partial_{x}^{\alpha} b(t, x, k),
$$

since function $\Psi$ in Theorem 8.4 vanishes. From this we can find amplitude $b$ from the discrete version of the transport equations, details of which we omit here. Finally, we note that we can also have an asymptotic expansion for the amplitude $b$ in (10.5) in terms of discrete differences $\triangle_{\xi}^{\alpha}$ and corresponding derivatives $\partial_{x}^{(\alpha)}$ instead of derivatives $\partial_{\xi}^{\alpha}$ and $\partial_{x}^{\alpha}$, respectively, if we use Remark 8.5 instead of Theorem 8.4.

\section{REFERENCES}

[1] M. S. Agranovich, Spectral properties of elliptic pseudodifferential operators on a closed curve. (Russian) Funktsional. Anal. i Prilozhen. 13 (1979), no. 4, 54-56.

[2] M. S. Agranovich, Elliptic pseudodifferential operators on a closed curve. (Russian) Trudy Moskov. Mat. Obshch. 47 (1984), 22-67, 246.

[3] B. A. Amosov, On the theory of pseudodifferential operators on the circle. (Russian) Uspekhi Mat. Nauk 43 (1988), 169-170; translation in Russian Math. Surveys 43 (1988), no. 3, 197-198.

[4] A. Boulkhemair, $L^{2}$ estimates for pseudodifferential operators. Ann. Scuola Norm. Sup. Pisa Cl. Sci. (4) 22 (1995), no. 1, 155-183.

[5] J. Bourgain, Exponential sums and nonlinear Schrödinger equations. Geom. Funct. Anal. 3 (1993), 157-178.

[6] J. Bourgain, Fourier transform restriction phenomena for certain lattice subsets and applications to nonlinear evolution equations. I. Schrödinger equations. Geom. Funct. Anal. 3 (1993), 107156.

[7] J. Bourgain, Global solutions of nonlinear Schrödinger equations. American Mathematical Society Colloquium Publications, 1999.

[8] A. P. Calderón and R. Vaillancourt, On the boundedness of pseudo-differential operators, J. Math. Soc. Japan 23 (1971), 374-378.

[9] R. R. Coifman and Y. Meyer, Au-delà des opérateurs pseudo-différentiels, Astérisque 57 (1978).

[10] H. O. Cordes, On compactness of commutators of multiplications and convolutions, and boundedness of pseudo-differential operators, J. Funct. Anal. 18 (1975), 115-131.

[11] J. Elschner, Singular ordinary differential operators and pseudodifferential equations. Lecture Notes in Mathematics, 1128. Springer-Verlag, Berlin, 1985.

[12] G. Garello, A. Morando, $L^{p}$-boundedness for pseudodifferential operators with non-smooth symbols and applications. Boll. Unione Mat. Ital. Sez. B Artic. Ric. Mat. (8) 8 (2005), 461-503.

[13] K. Gröchenig, Foundations of time-frequency analysis. Applied and Numerical Harmonic Analysis. Birkhäuser, 2001.

[14] L. Hörmander, The Analysis of Linear Partial Differential Operators IV. Springer-Verlag, 1985. 
[15] H. Kumano-go, M. Nagase, Pseudo-differential operators with non-regular symbols and applications. Funkcial. Ekvac. 21 (1978), no. 2, 151-192.

[16] W. McLean, Local and global description of periodic pseudodifferential operators. Math. Nachr. 150 (1991), 151-161.

[17] S. T. Melo, Characterizations of pseudodifferential operators on the circle. Proc. Amer. Math. Soc. 125 (1997), 1407-1412.

[18] S. Prössdorf, R. Schneider, Spline approximation methods for multidimensional periodic pseudodifferential equations. Integral Equations Operator Theory 15 (1992), no. 4, 626-672.

[19] M. Ruzhansky, M. Sugimoto, Global calculus of Fourier integral operators, weighted estimates, and applications to global analysis of hyperbolic equations, in Advances in pseudo-differential operators, 65-78, Oper. Theory Adv. Appl., 164, Birkhäuser, 2006.

[20] M. Ruzhansky and M. Sugimoto, Global $L^{2}$ boundedness theorems for a class of Fourier integral operators, Comm. Partial Differential Equations, 31 (2006), 547-569.

[21] M. Ruzhansky and M. Sugimoto, Weighted $L^{2}$ estimates for a class of Fourier integral operators, arXiv:0711.2868 1

[22] M. Ruzhansky, V. Turunen, On the Fourier analysis of operators on the torus, Modern trends in pseudo-differential operators, 87-105, Oper. Theory Adv. Appl., 172, Birkhäuser, Basel, 2007.

[23] M. Ruzhansky, V. Turunen, Global quantization of pseudo-differential operators on $\mathbb{S}^{3}$, preprint.

[24] M. Ruzhansky, V. Turunen, Pseudo-differential operators and symmetries, monograph in preparation, to appear in Birkhäuser.

[25] J. Saranen, G. Vainikko, Periodic integral and pseudodifferential equations with numerical approximation. Springer-Verlag, 2002.

[26] J. Saranen, W. L. Wendland, The Fourier series representation of pseudodifferential operators on closed curves. Complex Variables Theory Appl. 8 (1987), no. 1-2, 55-64.

[27] J. Sjöstrand, An algebra of pseudodifferential operators. Math. Res. Lett. 1 (1994), 185-192.

[28] E. M. Stein, Harmonic analysis: real-variable methods, orthogonality, and oscillatory integrals. Princeton University Press, Princeton, NJ, 1993.

[29] M. Sugimoto, Pseudo-differential operators on Besov spaces. Tsukuba J. Math. 12 (1988), no. $1,43-63$.

[30] M. E. Taylor, Noncommutative microlocal analysis. Mem. AMS 52 (1984), No. 313.

[31] V. Turunen, Commutator characterization of periodic pseudodifferential operators. Z. Anal. Anw. 19 (2000), 95-108.

[32] V. Turunen, G. Vainikko, On symbol analysis of periodic pseudodifferential operators. Z. Anal. Anw. 17 (1998), 9-22.

[33] G. M. Vainikko, I. K. Lifanov, Generalization and use of the theory of pseudodifferential operators in the modeling of some problems in mechanics. (Russian) Dokl. Akad. Nauk 373 (2000), no. 2, 157-160.

[34] G. M. Vainikko, I. K. Lifanov, The modeling of problems in aerodynamics and wave diffraction and the extension of Cauchy-type integral operators on closed and open curves. (Russian) Differ. Uravn. 36 (2000), no. 9, 1184-1195, 1293; translation in Differ. Equ. 36 (2000), no. 9, 1310-1322.

Michael Ruzhansky:

Department of Mathematics

IMPERIAL COLLEGE LONDON

180 QueEn's Gate, London SW7 2AZ

UNITED KINGDOM

E-mail address m.ruzhansky@imperial.ac.uk

Ville Turunen:

Helsinki University of TeChNOLOGY

Institute of Mathematics

P.O. Box 1100

FI-02015 HUT

FINLAND 
E-mail address ville.turunen@hut.fi 